\title{
Queue-Aware Energy-Efficient Joint Remote Radio Head Activation and Beamforming in Cloud Radio Access Networks
}

\author{
Jian Li, Jingxian Wu, Senior Member, IEEE, Mugen Peng ${ }^{\dagger}$, Senior Member, IEEE, Ping Zhang ${ }^{\dagger}$, Senior \\ Member, IEEE
}

\begin{abstract}
In this paper, we study the stochastic optimization of cloud radio access networks (C-RANs) by joint remote radio head (RRH) activation and beamforming in the downlink. Unlike most previous works that only consider a static optimization framework with full traffic buffers, we formulate a dynamic optimization problem by explicitly considering the effects of random traffic arrivals and time-varying channel fading. The stochastic formulation can quantify the tradeoff between power consumption and queuing delay. Leveraging on the Lyapunov optimization technique, the stochastic optimization problem can be transformed into a per-slot penalized weighted sum rate maximization problem, which is shown to be non-deterministic polynomial-time hard. Based on the equivalence between the penalized weighted sum rate maximization problem and the penalized weighted minimum mean square error (WMMSE) problem, the group sparse beamforming optimization based WMMSE algorithm and the relaxed integer programming based WMMSE algorithm are proposed to efficiently obtain the joint RRH activation and beamforming policy. Both algorithms can converge to a stationary solution with low-complexity and can be implemented in a parallel manner, thus they are highly scalable to large-scale C-RANs. In addition, these two proposed algorithms provide a flexible and efficient means to adjust the power-delay tradeoff on demand.
\end{abstract}

Index Terms-Cloud radio access networks (C-RANs), Lyapunov optimization, penalized weighted minimum mean square error (WMMSE), Lagrangian dual decomposition.

\section{INTRODUCTION}

The fifth-generation $(5 \mathrm{G})$ wireless networks are expected to provide ubiquitous services to a larger number of simultaneous mobile devices with device density far beyond the current wireless communication systems. To cope with these challenges, ultra-dense low power nodes and cloud computing are regarded as two of the most promising techniques [1]. Leveraged on low power node and cloud computing, the cloud radio access network (C-RAN), first proposed in [2], is expected to revolutionize the architecture and operations of future wireless systems, and it has attracted considerable amount of attentions in both academia and industry [3] [4]. As

Jian Li (e-mail: lijian.wspn@gmail.com), Mugen Peng (e-mail: pmg@bupt.edu.cn), Ping Zhang (e-mail:pzhang@bupt.edu.cn) are with the Key Laboratory of Universal Wireless Communications for Ministry of Education, Beijing University of Posts and Telecommunications, Beijing, 100876, China. Jingxian Wu (e-mail: wuj@uark.edu) is with the Department of Electrical Engineering, University of Arkansas, Fayetteville, 72701, USA.

Part of this work has been presented at IEEE Global Communications Conference (GLOBECOM), San Diego, CA, USA, Dec. 2015.

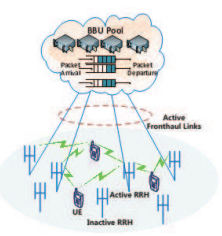

Fig. 1. Architecture of heterogeneous cloud radio access networks

shown in Fig. 11 a large number of remote radio heads (RRHs) are densely deployed in the space domain for C-RANs. Each $\mathrm{RRH}$ is configured only with the front radio frequency (RF) components and some basic transmission/reception functionalities. The RRHs are connected to the baseband unit (BBU) pool through high-bandwidth and low-latency fronthaul links to enable real-time cloud computing. The C-RANs can act as a platform for the practical implementation of coordinated multi-point (CoMP) transmission concepts [5]. Specifically, the BBU pool computes the beamforming weight coefficients for different RRHs, and sends the precoded data to various active RRHs. Then the active RRHs cooperatively transmit the precoded data to different UEs. The signals observed at each UE are superpositions of signals from multiple active RRHs. The beamforming weight coefficients are designed to steer the data to their intended receivers in the spatial domain. That is, for a given UE, the desired signals are combined coherently yet the interfering signals are combined out-of-phase. Here the joint beamforming aims to improve the signal-to-interferenceplus-noise ratio (SINR) in order to significantly improve the spectral efficiency of C-RANs.

Various beamforming designs have been studied for CoMP in C-RANs with different optimization objectives and constraints recently. In [6] and [7], the number of active front haul links is minimized under a SINR constraint for each user equipment (UE) and a power constraint for each RRH. The problem is solved by minimizing an approximate of the original combinatorial objective function. The works in 8 and [9] aim to jointly optimize the set of RRHs serving each user and the corresponding beamformers, under the constraint of front haul capacity. Specifically, power minimization is studied in [8] and sum rate maximization is considered in [9]. The design problems in [8] [9] fundamentally differ from that in [6] [7] in that they explicitly consider the fronthaul capacity. The problem of minimizing the overall power consumption and CoMP operational costs by joint user association 
and downlink beamforming was studied in [10], where the problem was addressed using a mixed integer second-order cone program framework. Similar problem has been studied in [11], where the user association and beamforming were considered in both downlink and uplink C-RANs. As existing solutions considering only the downlink like [10] cannot be modified in a straightforward way to solve the problem in [11], efficient algorithms were proposed utilizing the uplinkdownlink duality result.

The dense deployment of RRHs imposes new technical challenges for the design and implementation of large scale C-RANs. With the centralized processing at the BBU pool, the power consumed by the fronthaul links that provide highcapacity connections with BBU pool becomes comparable to that for transmission [12]. Therefore, in order to reduce power consumption of the entire network, we can reduce the number of active RRHs by putting some of the RRHs into the sleep mode. The fronthaul links of sleeping RRH will also be turned off to save power consumption. Therefore, the scheduling of RRH activation plays a critical role in the development of energy-efficient C-RANs. Related problem has been studied in [13], where two efficient group sparse beamforming algorithms were proposed to obtain the subset of active RRHs and their corresponding beamformers. The works in [14] compares the energy efficiencies of two different downlink transmission strategies in C-RANs by taking the RRH transmission power, RRH activation power, and load-dependent fronthaul power into considerations. Compared with the optimal exhaustive search method, the computational complexity of the algorithms in [6]-[11], [13], [14] can be significantly reduced, which, however, can still be very intensive for large-scale C-RANs. This is due to the fact that a series of convex problems (e.g. SDP, SOCP) have to be solved centrally using standard CVX solvers. Furthermore, the aforementioned literatures are typically based on snapshot-based static models, which indicates that the stochastic and time-varying features are not considered into the formulations. Therefore, only the physical layer performance metrics such as power and throughput are optimized and the resulting control policy is only adaptive to channel state information (CSI). In practice, delay is also a key metric to measure the quality-of-service $(\mathrm{QoS})$, which has also been neglected in these literatures. Intuitively, there is a fundamental tradeoff between power consumption and queuing delay [15], thus it is important to jointly consider power consumption and delay to balance their tradeoff and to meet various performance requirement in C-RANs.

In practice, the stochastic control and delay analysis are usually investigated from the queue stability perspective in a time-varying system using the Lyapunov optimization technique. Many existing literatures have focused on stochastic optimization for time-varying wireless networks. A fundamental approach to stochastic resource allocation and routing for heterogeneous data networks was presented in [16], where the flow control is crucial to ensure no network resources are wasted whenever the traffic rates are inside or outside the capacity region. The authors of [17] investigated stochastic control for wireless networks with finite buffers, where the joint flow control, routing, and scheduling algorithms can achieve high network utility and deterministically bounded backlogs inside the network. The delay analysis was conducted in [18] for suboptimal scheduling in one-hop wireless networks with general interference set constraints and time-correlated traffic arrivals. There also have been lots of works that focus on optimizing power under queue stability and interference constraints [19] [20]. However, these works generally adopt highly simplified physical-layer models for wireless channels, such as interference avoidance constraint or simple channelrate mapping function. They do not consider the complex non-linear relationship between signal power, interference power, and system throughput as in practical systems, which makes the system design much more challenging. This paper fundamentally differs from previous works in that we consider a power minimization problem by designing queue-aware joint optimization algorithms for C-RANs where both RRH activation set and beamforming vector are adaptive to queue state information and channel state information. In addition, the non-linear impacts of interence are explicitly quantified during the system design. Therefore, existing solutions can not be readily applied to the C-RAN setting considered in this paper.

Motivated by these facts, we propose to study dynamic joint RRH activation and beamforming for C-RANs by considering random traffic arrivals, queuing delays, and the time-varying fading channels. To the best of our knowledge, this is the first such work in C-RANs. Unlike the static optimization problems studied in the literatures, the problems considered in this paper are formulated as stochastic optimizations, which are notoriously difficult to solve but are important for practical systems. The main contributions of this paper are summarized as follows:

- The stochastic optimization of joint RRH activation and beamforming is developed for practical C-RANs. A wide range of detailed operations and constraints, such as beamforming, RRH activation, time-varying channel, random traffic arrivals, and fronthaul power consumption, are considered in the formulation. To the best of our knowledge, this is the first paper that achieves stochastic control of power and delay by considering realistic system operations across multiple protocol layers.

- To tackle the NP-hardness of the optimization problem, two low-complexity algorithms are proposed using the group sparse beamforming (GSB) approach and the relaxed integer programming (RIP) approach, respectively. Both algorithms can be implemented in a parallel manner with closed-form expressions, thus they are scalable to large-scale C-RANs.

- The delay and power performance of the two proposed algorithms are numerically evaluated. Significant performance gains are achieved by the proposed algorithms due to the fact that they are adaptive to the queue state information. The proposed algorithms can provide a flexible and efficient means to control the delay-power tradeoff on demand. All these make the proposed algorithms attractive and useful in practical applications.

The remainder of this paper is organized as follows. In 
Section II, we introduce the system model and formulate the stochastic optimization problem. In Section III, the Lyapunov optimization is introduced and utilized to obtain a penalized weighted sum rate maximization problem for each slot. The optimization problem is solved by two efficient algorithms based on GSB approach and RIP approach in Section IV and Section V, respectively. Numerical results are presented in Section VI. Finally, we conclude our paper in Section VII. The acronyms used in this paper are summarized in Table I.

TABLE I

SUMMARY OF ACRONYMS

\begin{tabular}{l|l}
\hline Acronym & Description \\
\hline C-RAN & cloud radio access network \\
BBU & baseband unit \\
RRH & remote radio head \\
MSE & mean square error \\
MMSE & minimum mean square error \\
WMMSE & weighted minimum mean square error \\
GSB & group sparse beamforming \\
RIP & relaxed integer programming \\
BCD & block coordinate descent \\
LASSO & least absolute shrinkage and selection operator \\
ADMM & alternating direction method of multipliers \\
FJP & full joint processing \\
\hline
\end{tabular}

Throughout this paper, lower-case bold letters denote vectors and and upper-case bold letters denote matrices. I denotes identity matrix. $\mathbb{C}$ denotes complex domain and the complex Gaussian distribution with mean $\mathbf{m}$ and covariance matrix $\mathbf{R}$ is represented by $\mathcal{C N}(\mathbf{m}, \mathbf{R}) . \mathbb{E}[\cdot]$ and $\operatorname{det}(\cdot)$ represent expectation and determinant operators, respectively. $\operatorname{Re}\{\cdot\}$ is the real part operator. $\|\cdot\|_{p}$ denotes $\ell_{p}$-norm of a vector. The inverse, transpose, conjugate transpose operators are denoted as $(\cdot)^{-1},(\cdot)^{T},(\cdot)^{H}$, respectively.

\section{SYSTEM MODEL}

\section{A. Scenario Description}

We consider a downlink C-RAN with $K$ RRHs and $I$ UEs, where each RRH is equipped with $M$ antennas and each UE has $N$ antennas. Let $\mathcal{K}$ and $\mathcal{I}$ denote the set of RRHs and the set of UEs, respectively. The bandwidth of the system is $W$. We also assume that the network operates in slotted time with time dimension partitioned into decision slots indexed by $t \in\{0,1,2, \ldots\}$ with slot duration $\tau$.

Let $\mathbf{H}_{k i}(t) \in \mathbb{C}^{N \times M}$ denote the channel state information (CSI) matrix from RRH $k$ to UE $i$ at slot $t$, let $\mathbf{H}_{i}(t)=\left[\mathbf{H}_{1 i}(t), \mathbf{H}_{2 i}(t), \ldots, \mathbf{H}_{K i}(t)\right] \in \mathbb{C}^{N \times M K}$ denote the CSI matrix from all RRHs to UE $i$ at slot $t$, and let $\mathbf{H}(t)=$ $\left[\mathbf{H}_{i}(t), \ldots, \mathbf{H}_{I}(t)\right] \in \mathbb{C}^{N \times M I K}$ denote the network CSI at slot $t$. The channel is assumed to follow quasi-static block fading, where each element of $\mathbf{H}(t)$ keeps constant for the duration of a slot, but is identically and independently distributed (i.i.d.) across different slots. Let $\mathbf{w}_{k i}(t) \in \mathbb{C}^{M \times 1}$ denote the beamforming vector at RRH $k$ for UE $i$ at slot $t$, let $\mathbf{w}_{i}(t)=$ $\left[\mathbf{w}_{1 i}^{T}(t), \ldots, \mathbf{w}_{K i}^{T}(t)\right]^{T} \in \mathbb{C}^{M K \times 1}$ denote the aggregated beamformer for UE $i$ at slot $t$, let $\tilde{\mathbf{w}}_{k}(t)=\left[\mathbf{w}_{k 1}^{T}(t), \ldots, \mathbf{w}_{k I}^{T}(t)\right]^{T} \in$ $\mathbb{C}^{M I \times 1}$ denote the aggregated beamformer used by RRH $k$ at slot $t$, and let $\mathbf{w}(t)=\left[\tilde{\mathbf{w}}_{1}^{T}(t), \ldots, \tilde{\mathbf{w}}_{K}^{T}(t)\right]^{T} \in \mathcal{C}^{M I K \times 1}$ denote the aggregated beamformer of the entire network at slot $t$.
Assume that each UE has its own data stream. Let $a_{i}(t)$ denote the data message for UE $i$ at slot $t$. Without loss of generality, we further assume that $\mathbb{E}\left[a_{i}^{2}(t)\right]=1$ and each $a_{i}(t)$ is i.i.d. among UEs. With linear beamforming operated centrally in the BBU pool, the baseband signal to be transmitted by RRH $k$ at slot $t$ is

$$
\mathbf{x}_{k}(t)=\sum_{i \in \mathcal{I}} \mathbf{w}_{k i}(t) a_{i}(t)
$$

The encoded baseband signal $\mathbf{x}_{k}(t)$ is delivered to RRH $k$ for radio transmission through corresponding fronthaul link. It is worth noting that as we focus on the issue of powerdelay tradeoff in this paper, we assume that the fronthaul links are provisioned with sufficiently high capacity and negligible latency 1 The signal observed by each UE is the superposition of signals from all RRHs. The received signal at UE $i$ is given by

$$
\mathbf{r}_{i}(t)=\mathbf{H}_{i}(t) \mathbf{w}_{i}(t) a_{i}(t)+\sum_{j \neq i} \mathbf{H}_{i}(t) \mathbf{w}_{j}(t) a_{j}(t)+\mathbf{z}_{i}(t)
$$

where $\mathbf{z}_{i}(t) \in \mathbb{C}^{N \times 1}$ is the additive white Gaussian noise (AWGN) at slot $t$ with distribution $\mathcal{C N}\left(0, \sigma^{2} \mathbf{I}\right)$. We assume that all the UEs adopt single user detection and the interference is treated as noise. The achievable data rate in the unit of $\mathrm{bps} / \mathrm{Hz}$ of $\mathrm{UE} i$ is given by

$$
\begin{aligned}
R_{i}(t)= & \log _{2} \operatorname{det}\left(\mathbf{I}+\mathbf{H}_{i}(t) \mathbf{w}_{i}(t) \mathbf{w}_{i}^{H}(t) \mathbf{H}_{i}^{H}(t)\right. \\
& \left.\left(\sum_{j \neq i} \mathbf{H}_{i}(t) \mathbf{w}_{j}(t) \mathbf{w}_{j}^{H}(t) \mathbf{H}_{i}^{H}(t)+\sigma^{2} \mathbf{I}\right)^{-1}\right) .
\end{aligned}
$$

\section{B. Network Power Consumption Model}

In C-RANs, the extensive use of high-capacity low-latency fronthaul links makes the fronthaul power consumption comparable to the transmission power of RRHs [22]. Here we consider the passive optical network to provide the effective high-capacity fronthaul connections between the RRHs and the BBU pool. The passive optical network consists of optical network units and an optical line terminal that connects a set of associated optical network units through a single optical fiber [23]. From the perspective of energy saving, some RRHs and their associated optical network units can be switched into sleep mode with negligible power consumption, but the optical line terminal with constant power consumption $P_{\mathrm{OLT}}$ cannot go into sleep mode as it plays the roles of distributer, aggregator and arbitrator of the transport network. Here we ignore $P_{\text {OLT }}$ because it is a constant and will not affect the scheduling and optimization results. Let $P_{k}^{\mathrm{ONU}}$ denote the constant power consumed by the optical network unit associated with active RRH $k$. Besides, due to the realtime $\mathrm{A} / \mathrm{D}$ and $\mathrm{D} / \mathrm{A}$ processing at each $\mathrm{RRH}$, static circuit power $P_{k}^{\mathrm{S}}$ is also consumed. Thus, the amount of static power consumption associated with RRH $k$ during active mode is $P_{k}^{\mathrm{c}}=P_{k}^{\mathrm{s}}+P_{k}^{\mathrm{ONU}}$. When RRH $k$ and its corresponding fronthaul link are switched into the sleep mode, there is no

\footnotetext{
${ }^{1}$ The impact of finite fronthaul capacity on fronthaul compression and quantization in C-RANs has been investigated recently in [21], which does not consider the power-delay tradeoff. It is expected that finite fronthaul capacity will negatively affect the performance of the proposed algorithm.
} 
static power consumption. Let $\mathcal{A}(t) \subseteq \mathcal{K}$ denote the set of active RRHs at slot $t$. The network power consumption at slot $t$ is given by

$$
p(\mathcal{A}(t), \mathbf{w}(t))=\sum_{k \in \mathcal{A}(t)}\left(\frac{1}{\eta_{k}}\left\|\tilde{\mathbf{w}}_{k}(t)\right\|_{2}^{2}+P_{k}^{c}\right),
$$

where $\eta_{k}$ is the drain efficiency of RF power amplifier at RRH $k$. Note that the load-dependent fronthaul power consumption model has been considered in [14], while its impact on our formulation will be left for future study. The network power consumption is a random process, in that it depends on the policy of RRH activation set and corresponding beamforming vectors, which is dynamically determined with the observation of traffic queues and channel conditions at each slot.

\section{Queue Stability and Problem Formulation}

The BBU pool maintains $I$ traffic queues for the random traffic arrivals towards $I$ UEs. Let $\mathbf{A}(t)=\left[A_{1}(t), \ldots, A_{I}(t)\right]$ be the vector of stochastic traffic data arrivals (bits) at the end of slot $t$. We assume that the traffic arrival $A_{i}(t)$ is independent w.r.t. $i$ and i.i.d. over slots according to a general distribution with mean $\mathbb{E}\left[A_{i}(t)\right]=\lambda_{i}$. Let $\mathbf{Q}(t)=\left[Q_{1}(t), \ldots, Q_{I}(t)\right]$ denote the vector of queue state information (QSI) (bits) for the $I$ UEs at the beginning of slot $t$. Therefore, the queue dynamic for UE $i$ is given by

$$
Q_{i}(t+1)=\max \left[Q_{i}(t)-\mu_{i}(t), 0\right]+A_{i}(t),
$$

where the amount of traffic departure at slot $t$ is given by $\mu_{i}(t)=W \tau R_{i}(t)$.

To model the impacts of joint RRH activation and beamforming policy on average queue delay and average network power consumption, we first present the definitions of queue stability, stability region and throughput optimal policy as follows [24].

Definition 1 (Queue Stability): A discrete time queue $Q(t)$ is strongly stable if

$$
\limsup _{T \rightarrow \infty} \frac{1}{T} \sum_{t=0}^{T-1} \mathbb{E}[Q(t)]<\infty .
$$

Furthermore, a network of queues is stable if all individual queues of the network are stable.

Definition 2: (Stability Region and Throughput-Optimal Policy): The stability region $\mathcal{C}$ is the closure of the set of all the arrival rate vectors $\boldsymbol{\lambda}=\left\{\lambda_{i}: i \in \mathcal{I}\right\}$ that can be stabilized in a C-RAN. A throughput-optimal resource optimization policy is a policy that stabilizes all the arrival rate vectors $\left\{\lambda_{i}: i \in \mathcal{I}\right\}$ within the stability region $\mathcal{C}$.

The objective is to simultaneously maintain the network queue stability and minimize the network power consumption, by using joint RRH activation and beamforming. The problem can be formulated as the following stochastic optimization problem:

$$
\begin{array}{ll}
\min . & \bar{p}=\lim _{T \rightarrow \infty} \frac{1}{T} \sum_{t=0}^{T-1} \mathbb{E}[p(\mathcal{A}(t), \mathbf{w}(t))] \\
\text { s.t. } & \mathrm{C} 1: \text { Queue } Q_{i}(t) \text { is strongly stable, } \forall i, \\
& \mathrm{C} 2:\left\|\tilde{\mathbf{w}}_{k}\right\|_{2}^{2} \leq P_{k},
\end{array}
$$

where the expectation $\mathbb{E}$ is taken with respect to the distribution of network power consumption, which depends on the random RRH activation set and beamforming vectors. $\mathrm{C} 1$ is the network stability constraint to guarantee a finite queue length for each queue. $\mathrm{C} 2$ is the constraint on the instantaneous per$\mathrm{RRH}$ power consumption. In practical C-RANs, the random traffic arrivals and the time-varying channel conditions are generally unpredictable. The stochastic nature of the channel conditions and traffic arrivals makes it impractical to calculate the optimal solution in an offline manner. To address this problem, we will resort to Lyapunov optimization, which can transform the stochastic optimization problem (7) into a deterministic one at each slot.

Remark 1: The queue stability constraint is used to depict and control the average delay. According to Definition 1, the queue stability is guaranteed if the average queue length is finite. Note that average delay is proportional to average queue length for a given traffic arrival rate from Little's Theorem. As suggested later in Section III, the average queue length can be arbitrarily bounded by choosing an appropriate control parameter.

\section{Problem TRansformation Based on Lyapunov OPTIMIZATION}

In this section, we will exploit the framework of Lyapunov optimization to solve the stochastic optimization problem in (7). Define the quadratic Lyapunov function as $L(\mathbf{Q}(t))=$ $\frac{1}{2} \sum_{i \in \mathcal{I}} Q_{i}(t)^{2}$, which serves as a scalar metric of queue congestion in the C-RAN. To keep the system stable by persistently pushing the Lyapunov function towards a lower congestion state, the one-step conditional Lyapunov drift is defined as

$$
\Delta(\mathbf{Q}(t))=\mathbb{E}[L(\mathbf{Q}(t+1))-L(\mathbf{Q}(t)) \mid \mathbf{Q}(t)],
$$

where $\mathbb{E}$ is the conditional expectation taken with respect to the distribution of Lyapunov drift given queue state $\mathbf{Q}(t)$. The Lyapunov drift-plus-penalty function is defined as

$$
\Delta(\mathbf{Q}(t))+V \mathbb{E}[p(\mathcal{A}(t), \mathbf{w}(t)) \mid \mathbf{Q}(t)]
$$

where $\mathbb{E}$ is the conditional expectation taken with respect to the distribution of network power consumption given queue state $\mathbf{Q}(t)$, and $V>0$ represents an arbitrary control parameter. The parameter $V$ can be used to control the power-delay tradeoff. A larger $V$ means more emphasis will be put on power minimization during the optimization. On the other hand, when $V$ is small, queue stability carries more weight during the optimization. Suppose that the expectation of the penalty process $p(\mathcal{A}(t), \mathbf{w}(t))$ is deterministically bounded by some finite constant $p_{\min }, p_{\max }$, i.e. $p_{\min } \leq \mathbb{E}[p(\mathcal{A}(t), \mathbf{w}(t))] \leq p_{\max }$. Let $p^{*}$ denote the theoretical optimal value of (7), then the relationship between the Lyapunov drift-plus-penalty function and queue stability is established in Theorem 1 [24].

Theorem 1 (Lyapunov Optimization): Suppose there exist positive constants $B, \epsilon$ and $V$ such that for all slots $t \in$ $\{0,1,2, \ldots\}$ and all possible values of $\mathbf{Q}(t)$, the Lyapunov 
drift-plus-penalty function satisfies:

$\Delta(\mathbf{Q}(t))+V \mathbb{E}[p(\mathcal{A}(t), \mathbf{w}(t)) \mid \mathbf{Q}(t)] \leq B+V p^{*}-\epsilon \sum_{i=1}^{I} Q_{i}(t)$,

then all queues $Q_{i}(t)$ are strongly stable. The average queue length satisfies

$$
\limsup _{T \rightarrow \infty} \frac{1}{T} \sum_{t=0}^{T-1} \sum_{i=1}^{I} \mathbb{E}\left[Q_{i}(t)\right] \leq \frac{B+V\left(p^{*}-p_{\min }\right)}{\epsilon},
$$

and the average penalty of power consumption satisfies

$$
\limsup _{T \rightarrow \infty} \frac{1}{T} \sum_{t=0}^{T-1} \mathbb{E}[p(\mathcal{A}(t), \mathbf{w}(t))] \leq p^{*}+\frac{B}{V} .
$$

Proof: The proof can follow that for Theorem 4.2 in [24].

The results in Theorem 1 motivate us to minimize the Lyapunov drift-plus-penalty in (9) to achieve the maximum queue stability region and obtain throughput-optimal policy. Rather than directly minimize (9), our policy actually seeks to minimize the upper bound of (9), which is given by the following lemma [Lemma 4.6 of 23].

Lemma 1 (Upper Bound of Lyapunov Drift-plus-penalty): Under any control policy, the drift-plus-penalty has the following upper bound for all $t$, all possible values of $\mathbf{Q}(\mathbf{t})$ and all parameters $V>0$,

$$
\begin{aligned}
& \Delta(\mathbf{Q}(t))+V \mathbb{E}[p(\mathcal{A}(t), \mathbf{w}(t)) \mid \mathbf{Q}(t)] \leq B+ \\
& V \mathbb{E}[p(\mathcal{A}(t), \mathbf{w}(t)) \mid \mathbf{Q}(t)]+\sum_{i \in \mathcal{I}} Q_{i}(t) \mathbb{E}\left[A_{i}(t)-\mu_{i}(t) \mid \mathbf{Q}(t)\right],
\end{aligned}
$$

where $B$ is a positive constant and for all slot $t$ satisfies $B \geq$ $\frac{1}{2} \sum_{i=1}^{I} \mathbb{E}\left[A_{i}^{2}(t)+\mu_{i}^{2}(t) \mid \mathbf{Q}(t)\right]$.

Proof: The proof is in Appendix A.

By the principle of opportunistically minimizing an expectation [24], the policy that minimizes $\mathbb{E}[f(t) \mid \mathbf{Q}(t)]$ is the one that minimizes $f(t)$ with the observation of $\mathbf{Q}(t)$. Besides, neither $\sum_{i \in \mathcal{I}} Q_{i}(t) A_{i}(t)$ nor $B$ in 13 will be affected by the policy at slot $t$. Therefore, the optimization problem can be simplified to

$$
\max _{\mathcal{A}(t), \mathbf{w}(t)} \sum_{i \in \mathcal{I}} Q_{i}(t) \mu_{i}(t)-V p(\mathcal{A}(t), \mathbf{w}(t)) .
$$

The following theorem justifies the throughput optimality by solving problem (14) optimally.

Theorem 2: The RRH activation $\mathcal{A}(t)$ and beamforming $\mathbf{w}(t)$ given by solving (14) optimally achieves the maximum stability region $\mathcal{C}$ in $\mathrm{C}$-RANs.

Proof: The proof is in Appendix B.

However, the weighted sum rate term in (14) is nonconvex and is shown to be NP-hard in wireless networks with interference [26]. It is thus extremely difficult, if not impossible, to get the globally optimal solution to (14) through efficient algorithms in polynomial time. Rather than seeking global optimality, we will focus on developing low-complexity algorithms that produce suboptimal solutions to (14). The following theorem characterizes the performance of (14) under suboptimal solutions.

Theorem 3: Let $\phi$ and $C$ be constants such that $0<\phi \leq 1$ and $C \geq 0$. Suppose there is an $\epsilon>0$, such that

$$
\boldsymbol{\lambda}+\epsilon \mathbf{1} \in \phi \mathcal{C} .
$$

If the suboptimal solution makes (possibly randomized) decisions every slot to satisfy

$$
\sum_{i=1}^{I} Q_{i}(t) \mathbb{E}\left[\mu_{i}(t) \mid \mathbf{Q}(t)\right] \geq \phi\left(\max \sum_{i=1}^{I} Q_{i}(t) \mu_{i}\right)-C,
$$

then the network is strongly stable.

Proof: The proof can follow that for Theorem 6.3 in [24].

Theorem 3 suggests that the suboptimal solutions that satisfy (16) can provide stability whenever the traffic arrival rates are interior to a $\phi$-scaled version of the stability region. In this paper, we will develop suboptimal solutions by relaxing and reformulating the optimization problem in (14), while it is extremely difficult to quantify the $\phi$ and $C$ that the algorithms can achieve. The stability region analysis for our proposed algorithms remains challenging and is left for future work.

Remark 2: For the general case that the arrival rate vector is outside the stability region $\mathcal{C}$ or the possible reduced one $\phi \mathcal{C}$, congestion controls are need to constrain the arrival rate vector into the stability region. In this case, the problem can be decomposed into a congestion control subproblem and joint RRH activation and beamforming subproblem by following the framework in [25]. By doing so, we can have the separate congestion control subproblem and joint RRH activation and beamforming subproblem, and the deterministic worst-case delay bound can be guaranteed for each traffic queue.

\section{GSB-based Equivalent Penalized WMMSE ALGORITHM}

In this section, we will use the GSB approach to solve the optimization problem in (14).

\section{A. Group Sparse Beamforming Formulation}

Since only a subset RRHs will be active, we can solve the problem by exploiting the group sparse structure of the aggregated beamforming vector $\mathbf{w}(t)=\left[\tilde{\mathbf{w}}_{1}^{T}(t), \cdots, \tilde{\mathbf{w}}_{K}^{T}(t)\right]^{T} \in$ $\mathcal{C}^{M I K \times 1}$, where the coefficients in $\tilde{\mathbf{w}}_{k}^{T}(t)$ form a group [27]. When the RRH $k$ is switched off, all the coefficients in the vector $\tilde{\mathbf{w}}_{k}$ are 0 , which results in the group sparse structure. The mixed $\ell_{1} / \ell_{p}$-norm is shown to be effective to induce group sparsity and has attracted lots of attentions [28]. In this subsection, we try to construct a convex relaxation of (4), resulting in a weighted mixed $\ell_{1} / \ell_{2}$-norm. Specifically, we first calculate the tightest positively homogeneous lower bound of $p(\mathbf{w})$ with the definition $p_{h}(\mathbf{w})=\inf _{\phi>0} \frac{p(\phi \mathbf{w})}{\phi}, 0<\phi<\infty$, which is still nonconvex. We then calculate the Fenchel conjugate to provide its convex envelope $\hat{p}(\mathbf{w})$, which is called as the tightest convex positively homogeneous lower bound of $p(\mathbf{w})$ and is given by the following proposition. 
Proposition 1: The tightest convex positively homogeneous lower bound of (4) is given by

$$
\hat{p}(\mathbf{w}(t))=2 \sum_{k \in \mathcal{K}} \sqrt{\frac{P_{k}^{c}}{\eta_{k}}}\left\|\tilde{\mathbf{w}}_{k}(t)\right\|_{2},
$$

which is a weighted mixed $\ell_{1} / \ell_{2}$-norm.

Proof: The proof is in Appendix C.

The above proposition indicates that the mixed $\ell_{1} / \ell_{2}$-norm can provide a convex relaxation for the cost function (4), thus it can further introduce group sparsity to w, that is, many subvectors, $\tilde{\mathbf{w}}_{k}$ will be 0 , which corresponds to inactive RRHs. While the active set of RRHs corresponds to the non-zero subvectors in $\mathbf{w}$. By minimizing the weighted mixed $\ell_{1} / \ell_{2}$-norm (17) of $\mathbf{w}$, the zero entries of $\mathbf{w}$ will be made to align to the same group $\tilde{\mathbf{w}}_{k}$, such that the corresponding RRH is forced to switch off. The weight for each group embraces additional system parameters. Intuitively, the RRH with a higher static power consumption and a lower RF power amplifier drain efficiency will have a high priority being forced to switch off.

Using the weighted mixed $\ell_{1} / \ell_{2}$-norm as a surrogate objective function in (14), we finally have the following queueaware group sparse beamforming problem:

$$
\begin{aligned}
& \max _{\mathbf{w}} . \sum_{i \in \mathcal{I}} Q_{i} R_{i}-\sum_{k \in \mathcal{K}} \frac{2 V \sqrt{P_{k}^{c} / \eta_{k}}}{W \tau}\left\|\tilde{\mathbf{w}}_{k}\right\|_{2}, \\
& \text { s.t. }\left\|\tilde{\mathbf{w}}_{k}\right\|_{2}^{2} \leq P_{k} .
\end{aligned}
$$

In the above formulation, the slot index $t$ is skipped to simplify the notation.

Remark 3: The objective function in 18 is a convex relaxation to the original problem (14) using the group sparsity inducing norm. It has been shown in Proposition 1 that 18 is the tightest convex positively homogeneous lower bound of (14), that is, among all convex positively homogeneous functions, (18) has the smallest gap with 14. It is very challenging to quantify the exact performance gap, which normally requires specific prior information, e.g., in compressive sensing, the sparse signal is assumed to obey a power law (see Eq. (1.8) in [29]). However, our problem fundamentally differs from the existing compressive sensing problems in that we do not have any prior information about the optimal solution. The optimality analysis of the queue-aware group sparse beamforming algorithm will be left to our future work.

Next we will design a computationally efficient algorithm that produces a stationary solution to 18 by introducing an equivalent formulation.

\section{B. Equivalent Formulation and Penalized WMMSE algorithm}

The equivalence between weighted sum rate maximization problem and WMMSE problem is first established in [30] for multiple-input and multiple-output (MIMO) broadcast channel and generalized to MIMO interfering channel in [31]. By extending the equivalence in [30] [31], the penalized weighted sum rate maximization problem is equivalent to the following penalized WMMSE problem,

$$
\begin{aligned}
& \min _{\boldsymbol{\alpha}, \mathbf{u}, \mathbf{w}} \sum_{i \in \mathcal{I}} Q_{i}\left(\alpha_{i} e_{i}-\log \alpha_{i}\right)+\sum_{k \in \mathcal{K}} \beta_{k}\left\|\tilde{\mathbf{w}}_{k}\right\|_{2}, \\
& \text { s.t. }\left\|\tilde{\mathbf{w}}_{k}\right\|_{2}^{2} \leq P_{k},
\end{aligned}
$$

where $\boldsymbol{\alpha}=\left\{\alpha_{i} \mid i \in \mathcal{I}\right\}$ is the set of non-negative mean squared error (MSE) weights, $e_{i}=\mathbf{u}_{i}^{H}\left(\sum_{j \in \mathcal{I}} \mathbf{H}_{i} \mathbf{w}_{j} \mathbf{w}_{j}^{H} \mathbf{H}_{i}^{H}+\sigma^{2} \mathbf{I}\right) \mathbf{u}_{i}-$ $2 \operatorname{Re}\left\{\mathbf{u}_{i}^{H} \mathbf{H}_{i} \mathbf{w}_{i}\right\}+1$ is the MSE for estimating $s_{i}, \mathbf{u}=\left\{\mathbf{u}_{i} \in\right.$ $\left.\mathbb{C}^{N \times 1} \mid i \in \mathcal{I}\right\}$ is the collection of the receiving vectors for all UEs, and $\beta_{k}=\frac{2 V \sqrt{P_{k}^{c} / \eta_{k}}}{\log _{2}^{e} W \tau}$ is the parameter that will affect the number of active RRHs.

It is worth noting that problem 19] is not jointly convex in $\boldsymbol{\alpha}, \mathbf{u}, \mathbf{w}$, while it is convex with respect to each of the individual optimization variables when fixing the others. To this end, the block coordinate descent (BCD) method is utilized to obtain the stationary point of problem (19). As proven in [30], once the iterative process converges to a fixed point of problem (19), the fixed point is also a stationary point of the problem (18). It should be noted that the stationary point of problem (18) or 19 might not be globally optimal.

Under fixed $\mathbf{w}$ and $\boldsymbol{\alpha}$, minimizing the weighted sum-MSE leads to the well-known MMSE receiver:

$$
\mathbf{u}_{i}=\left(\sum_{j \in \mathcal{I}} \mathbf{H}_{i} \mathbf{w}_{j} \mathbf{w}_{j}^{H} \mathbf{H}_{i}^{H}+\sigma^{2} \mathbf{I}\right)^{-1} \mathbf{H}_{i} \mathbf{w}_{i} .
$$

With the MMSE receiver, the MSE $e_{i}$ can be written as

$$
e_{i}=1-\mathbf{w}_{i}^{H} \mathbf{H}_{i}^{H}\left(\sum_{j \in \mathcal{I}} \mathbf{H}_{i} \mathbf{w}_{j} \mathbf{w}_{j}^{H} \mathbf{H}_{i}^{H}+\sigma^{2} \mathbf{I}\right)^{-1} \mathbf{H}_{i} \mathbf{w}_{i}
$$

Under fixed $\mathbf{w}$ and $\mathbf{u}$, the closed-form $\boldsymbol{\alpha}$ can be obtained as follows according to the first-order optimality conditions:

$$
\alpha_{i}=e_{i}^{-1} \text {. }
$$

Under fixed $\mathbf{u}$ and $\boldsymbol{\alpha}$, the optimal $\mathbf{w}$ can be obtained by solving the following convex problem:

$$
\begin{aligned}
& \min _{\mathbf{w}} \cdot \sum_{i \in \mathcal{I}} \mathbf{w}_{i}^{H} \mathbf{C} \mathbf{w}_{i}-2 \sum_{i \in \mathcal{I}} \operatorname{Re}\left\{\mathbf{d}_{i}^{H} \mathbf{w}_{i}\right\}+\sum_{k \in \mathcal{K}} \beta_{k}\left\|\tilde{\mathbf{w}}_{k}\right\|_{2}, \\
& \text { s.t. }\left\|\tilde{\mathbf{w}}_{k}\right\|_{2}^{2} \leq P_{k},
\end{aligned}
$$

where $\mathbf{C}=\sum_{j \in \mathcal{I}} Q_{j} \alpha_{j} \mathbf{H}_{j}^{H} \mathbf{u}_{j} \mathbf{u}_{j}^{H} \mathbf{H}_{j}$ and $\mathbf{d}_{i}=Q_{i} \alpha_{i} \mathbf{H}_{i}^{H} \mathbf{u}_{i}$.

The algorithm is summarized in Algorithm 1 .

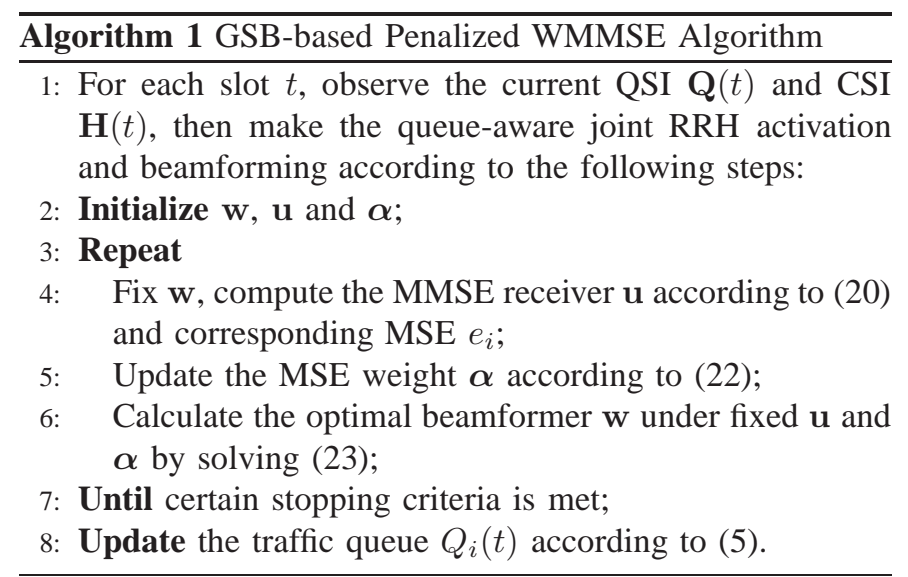

The objective function in (23) contains two parts: the quadratic part $\sum_{i \in \mathcal{I}} \mathbf{w}_{i}^{H} \mathbf{C} \mathbf{w}_{i}-2 \sum_{i \in \mathcal{I}} \operatorname{Re}\left\{\mathbf{d}_{i}^{H} \mathbf{w}_{i}\right\}$, and the $\ell_{2^{-}}$ norm part $\sum_{k \in \mathcal{K}} \beta_{k}\left\|\tilde{\mathbf{w}}_{k}\right\|_{2}$. Unlike the objective of standard 
group least absolute shrinkage and selection operator (LASSO) problem, the two parts are functions of different variables, i.e., $\mathbf{w}_{i}$ and $\tilde{\mathbf{w}}_{k}$, rather than the same variable. Therefore, existing computationally efficient algorithms developed for group LASSO in [32] cannot be readily applied to solve our modified group LASSO problem 23. This fact motivates us to find a new approach to solve the problem in 23. Our approach is based on the famous ADMM algorithm, which will be briefly reviewed below.

\section{Review of ADMM algorithm}

The ADMM algorithm, originally introduced in the 1970s, is a simple but powerful algorithm that is well suited to distributed convex optimization, and arbitrary-scale convex optimization. Specifically, the ADMM is designed to solve the following structured convex problem [33]

$$
\begin{array}{rl}
\min _{\mathbf{x} \in \mathbb{C}^{n}, \mathbf{z} \in \mathbb{C}^{m}} & f(\mathbf{x})+g(\mathbf{z}), \\
\text { s.t. } & \mathbf{A} \mathbf{x}+\mathbf{B} \mathbf{z}=\mathbf{c}, \\
& \mathbf{x} \in \mathcal{C}_{1}, \mathbf{z} \in \mathcal{C}_{2},
\end{array}
$$

where $\mathbf{A} \in \mathbb{C}^{k \times n}, \mathbf{B} \in \mathbb{C}^{k \times m}, \mathbf{c} \in \mathbb{C}^{k}, f(\cdot)$ and $g(\cdot)$ are convex functions, and $\mathcal{C}_{1}$ and $\mathcal{C}_{2}$ are non-empty convex sets. Correspondingly, the partial augmented Lagrangian function is given by

$$
\begin{aligned}
L_{\rho}(\mathbf{x}, \mathbf{z}, \mathbf{y}) & =f(\mathbf{x})+g(\mathbf{z})+\operatorname{Re}\left(\mathbf{y}^{H}(\mathbf{A x}+\mathbf{B} \mathbf{z}-\mathbf{c})\right) \\
& +\frac{\rho}{2}\|\mathbf{A} \mathbf{x}+\mathbf{B} \mathbf{z}-\mathbf{c}\|_{2}^{2},
\end{aligned}
$$

where $\mathbf{y} \in \mathbb{C}^{k}$ is the vector of Lagrangian dual variables associated with the linear equality constraint, and $\rho>0$ is a constant. The ADMM algorithm consists of the following iterations:

$$
\begin{gathered}
\mathbf{x}^{(n+1)}=\arg \min _{\mathbf{x}} L_{\rho}\left(\mathbf{x}, \mathbf{z}^{(n)}, \mathbf{y}^{(n)}\right), \\
\mathbf{z}^{(n+1)}=\arg \min _{\mathbf{z}} L_{\rho}\left(\mathbf{x}^{(n+1)}, \mathbf{z}, \mathbf{y}^{(n)}\right), \\
\mathbf{y}^{(n+1)}=\mathbf{y}^{(n)}+\rho\left(\mathbf{A} \mathbf{x}^{(n+1)}+\mathbf{B} \mathbf{z}^{(n+1)}-\mathbf{c}\right) .
\end{gathered}
$$

It can be easily seen that the ADMM algorithm takes the form of a decomposition-coordination procedure, in which the solutions to small local sub-problems are coordinated through dual variable update 28 to find a solution to a large global problem. Furthermore, the convergence is established in the following theorem [33].

Theorem 4: Assume that $\mathbf{A}^{T} \mathbf{A}$ and $\mathbf{B}^{T} \mathbf{B}$ are invertible, and the optimal solution to (24) exists. Then the updated sequence $\left\{\mathbf{x}^{(n)}, \mathbf{z}^{(n)}, \mathbf{y}^{(n)}\right\}$ is bounded and the converged $\left\{\mathbf{x}^{(n)}, \mathbf{z}^{(n)}\right\}$ is an optimal solution of (24).

\section{ADMM Algorithm to (23)}

In this subsection, based on splitting the variables $\mathbf{w}$, the tightly coupled large-sized problem in 23) will be decomposed into several sub-problems and solved efficiently by the ADMM algorithm with closed-form solutions.

To account for the difference between $\mathbf{w}_{i}$ and $\tilde{\mathbf{w}}_{k}$ in 23, we introduce a copy $\tilde{\mathbf{v}}_{k}$ for the original beamformer $\tilde{\mathbf{w}}_{k}$, and define $\mathbf{v}=\left[\tilde{\mathbf{v}}_{1}^{T}, \ldots, \tilde{\mathbf{v}}_{K}^{T}\right]^{T} \in \mathbb{C}^{M K I \times 1}$. The optimization problem in 23) can be equivalently expressed as

$$
\begin{aligned}
& \min _{\mathbf{w}, \mathbf{v}} \cdot \sum_{i \in \mathcal{I}} \mathbf{w}_{i}^{H} \mathbf{C w}_{i}-2 \sum_{i \in \mathcal{I}} \operatorname{Re}\left\{\mathbf{d}_{i}^{H} \mathbf{w}_{i}\right\}+\sum_{k \in \mathcal{K}} \beta_{k}\left\|\tilde{\mathbf{v}}_{k}\right\|_{2}, \\
& \text { s.t. }\left\|\tilde{\mathbf{v}}_{k}\right\|_{2}^{2} \leq P_{k}, \\
& \quad \tilde{\mathbf{v}}_{k}=\tilde{\mathbf{w}}_{k} .
\end{aligned}
$$

The partial augmented Lagrangian function of the above problem is given by

$$
\begin{aligned}
L(\mathbf{w}, \mathbf{v}, \mathbf{y}) & =\min _{\mathbf{w}, \mathbf{v}} \cdot \sum_{i \in \mathcal{I}} \mathbf{w}_{i}^{H} \mathbf{C} \mathbf{w}_{i}-2 \sum_{i \in \mathcal{I}} \operatorname{Re}\left\{\mathbf{d}_{i}^{H} \mathbf{w}_{i}\right\} \\
& +\sum_{k \in \mathcal{K}} \beta_{k}\left\|\tilde{\mathbf{v}}_{k}\right\|_{2}+\sum_{k \in \mathcal{K}} \operatorname{Re}\left\{\tilde{\mathbf{y}}_{k}^{H}\left(\tilde{\mathbf{v}}_{k}-\tilde{\mathbf{w}}_{k}\right)\right\} \\
& +\frac{\rho}{2} \sum_{k \in \mathcal{K}}\left\|\tilde{\mathbf{v}}_{k}-\tilde{\mathbf{w}}_{k}\right\|_{2}^{2}
\end{aligned}
$$

where $\mathbf{y}=\left[\tilde{\mathbf{y}}_{1}^{T}, \ldots, \tilde{\mathbf{y}}_{K}^{T}\right]^{T}$, with $\tilde{\mathbf{y}}_{k}=\left[\mathbf{y}_{k 1}^{T}, \ldots, \mathbf{y}_{k I}^{T}\right]^{T} \in$ $\mathbb{C}^{M I \times 1}$ being the vector of Lagrangian dual variables for the equality constraints in (29), and $\rho>0$ is some constant. The main steps of the ADMM algorithm are summarized in Algorithm 2]

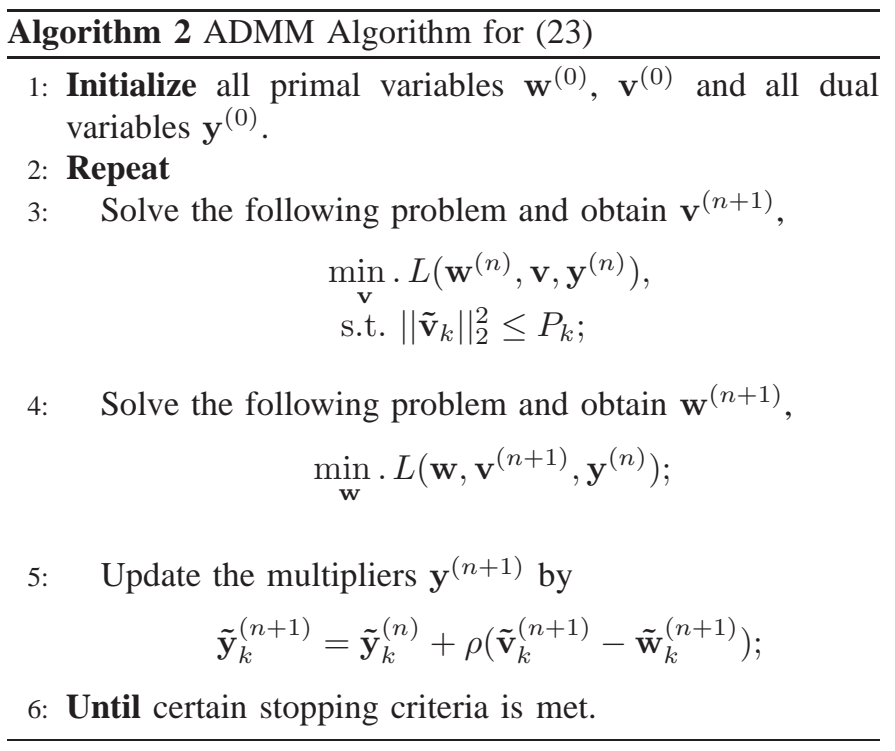

Before obtaining the closed-form expressions for each iteration in the above algorithm, the convergence of Algorithm 2 is first discussed.

Theorem 5: Every limit point $\mathbf{w}^{(n)}$ and $\mathbf{v}^{(n)}$ generated by Algorithm 2 is an optimal solution of problem (23).

Proof: The proof is in Appendix D.

In Algorithm 2, given $\mathbf{w}$ and $\mathbf{y}$, the step to obtain $\mathbf{v}$ can be further decomposed into $K$ sub-problems, each of which is associated with a RRH and can be solved in a parallel manner. By completing the squares in (30), the $k$-th convex sub-problem for RRH $k$ can be simplified to

$$
\begin{aligned}
& \min _{\tilde{\mathbf{v}}_{k}} . \beta_{k}\left\|\tilde{\mathbf{v}}_{k}\right\|_{2}+\frac{\rho}{2}\left\|\tilde{\mathbf{v}}_{k}-\tilde{\mathbf{w}}_{k}+\tilde{\mathbf{y}}_{k} / \rho\right\|_{2}^{2}, \\
& \text { s.t. }\left\|\tilde{\mathbf{v}}_{k}\right\|_{2}^{2} \leq P_{k} .
\end{aligned}
$$

The corresponding Karush-Kuhn-Tucker (KKT) conditions are given by

$$
\rho \mathbf{b}_{k}-\left(\rho+2 \gamma_{k}^{*}\right) \tilde{\mathbf{v}}_{k}^{*} \in \beta_{k} \partial\left(\left\|\tilde{\mathbf{v}}_{k}^{*}\right\|_{2}\right),
$$




$$
\left\|\tilde{\mathbf{v}}_{k}^{*}\right\|_{2}^{2} \leq P_{k}, \gamma_{k}^{*} \geq 0,\left(\left\|\tilde{\mathbf{v}}_{k}^{*}\right\|_{2}^{2}-P_{k}\right) \gamma_{k}^{*}=0,
$$

where $\mathbf{b}_{k}=\tilde{\mathbf{w}}_{k}-\tilde{\mathbf{y}}_{k} / \rho, \gamma_{k}^{*}$ is the optimal Lagrangian multiplier associated with the power constraint, and $\partial\left(\left\|\tilde{\mathbf{v}}_{k}^{*}\right\|_{2}\right)$ represents the subdifferential of $\ell_{2}$-norm $\|\cdot\|_{2}$ at the point $\tilde{\mathbf{v}}_{k}^{*}$, which can be expressed as follows [34]

$$
\partial\left(\left\|\tilde{\mathbf{v}}_{k}^{*}\right\|_{2}\right)=\left\{\begin{array}{ll}
\frac{\tilde{\mathbf{v}}_{k}^{*}}{\left\|\tilde{\mathbf{v}}_{k}^{*}\right\|_{2}}, & \tilde{\mathbf{v}}_{k}^{*} \neq 0 \\
\left\{\mathbf{x},\|\mathbf{x}\|_{2} \leq 1\right\}, & \tilde{\mathbf{v}}_{k}^{*}=0
\end{array} .\right.
$$

Therefore, we have $\tilde{\mathbf{v}}_{k}^{*}=0$ whenever $\left\|\rho \mathbf{b}_{k}\right\|_{2} \leq \beta_{k}$. When $\left\|\rho \mathbf{b}_{k}\right\|_{2}>\beta_{k}$, we have

$$
\tilde{\mathbf{v}}_{k}^{*}=\frac{\left(\rho\left\|\mathbf{b}_{k}\right\|_{2}-\beta_{k}\right) \mathbf{b}_{k}}{\left(\rho+2 \gamma_{k}^{*}\right)\left\|\mathbf{b}_{k}\right\|_{2}} .
$$

Furthermore, according to the complementary conditions, $\gamma_{k}^{*}=0$ if $\left\|\frac{\left(\rho\left\|\mathbf{b}_{k}\right\|_{2}-\beta_{k}\right) \mathbf{b}_{k}}{\rho\left\|\mathbf{b}_{k}\right\|_{2}}\right\|_{2}^{2}<P_{k}$. Otherwise, we should have $\left\|\frac{\left(\rho\left\|\mathbf{b}_{k}\right\|_{2}-\beta_{k}\right) \mathbf{b}_{k}}{\left(\rho+2 \gamma_{k}^{*}\right)|| \mathbf{b}_{k} \|_{2}}\right\|_{2}^{2}=P_{k}$, which indicates that $\gamma_{k}^{*}=$ $\frac{\rho|| \mathbf{b}_{k} \|_{2}-\beta_{k}-\rho \sqrt{P_{k}}}{2 \sqrt{P_{k}}}$. In a summary, the closed-form solution for updating $\mathbf{v}$ is given by

$$
\tilde{\mathbf{v}}_{k}^{*}= \begin{cases}\mathbf{0}, & \left\|\mathbf{b}_{k}\right\|_{2} \leq \frac{\beta_{k}}{\rho}, \\ \frac{\left(\rho\left\|\mathbf{b}_{k}\right\|_{2}-\beta_{k}\right) \mathbf{b}_{k}}{\rho \| \mathbf{b}_{k}||_{2}}, & \frac{\beta_{k}}{\rho}<\left\|\mathbf{b}_{k}\right\|_{2}<\frac{\beta_{k}}{\rho}+\sqrt{P_{k}}, \\ \frac{\mathbf{b}_{k} \sqrt{P_{k}}}{\left\|\mathbf{b}_{k}\right\|_{2}}, & \text { otherwise. }\end{cases}
$$

As for the step to obtain $\mathbf{w}$, it can be further decomposed into $I$ sub-problems, each of which is associated with a UE and can be solved in a parallel manner. By completing the squares in (30), the $i$-th unconstrained convex sub-problem for UE $i$ can be simplified as

$$
\min _{\mathbf{w}_{i}} \cdot \mathbf{w}_{i}^{H} \mathbf{C} \mathbf{w}_{i}-2 \operatorname{Re}\left\{\mathbf{d}_{i}^{H} \mathbf{w}_{i}\right\}+\frac{\rho}{2} \sum_{k \in \mathcal{K}}\left\|\tilde{\mathbf{w}}_{k}-\tilde{\mathbf{v}}_{k}-\tilde{\mathbf{y}}_{k} / \rho\right\|_{2}^{2} .
$$

Since the summation term in (37) is the summation of squared $\ell_{2}$-norms, it can be alternatively expressed as $\sum_{k \in \mathcal{K}}\left\|\tilde{\mathbf{w}}_{k}-\tilde{\mathbf{v}}_{k}-\tilde{\mathbf{y}}_{k} / \rho\right\|_{2}^{2}=\sum_{i \in \mathcal{I}}\left\|\mathbf{w}_{i}-\mathbf{v}_{i}-\mathbf{y}_{i} / \rho\right\|_{2}^{2}$. Differentiating the objective with respect to $\mathbf{w}_{i}$ and setting the result equal to zero, the optimal $\mathbf{w}_{i}$ is given by the following closedform expression:

$$
\mathbf{w}_{i}^{*}=(2 \mathbf{C}+\rho \mathbf{I})^{-1}\left(2 \mathbf{d}_{i}+\rho \mathbf{v}_{i}+\mathbf{y}_{i}\right),
$$

where $\mathbf{y}_{i}=\left[\mathbf{y}_{1 i}^{T}, \ldots, \mathbf{y}_{K i}^{T}\right]^{T} \in \mathbb{C}^{M K \times 1}$, with $\mathbf{y}_{k i} \in \mathbb{C}^{M \times 1}$ being the $i$-th block of $\tilde{\mathbf{y}}_{k}$.

\section{E. Discussions on Implementation and Complexity}

1) Further Reduction of the Number of Active RRHs: To further decrease the number of active RRHs by enhancing the group-sparsity for the beamformer, the reweighting procedure that adaptively reweights the coefficient $\beta_{k}$ in (23) can be utilized. Specifically, this can be done in step 6 of Algorithm 1 by solving problem (23) and updating the coefficient $\beta_{k}$ iteratively, see Section V-C in [13] for details. By doing so, the number of active RRHs is expected to be smaller than that obtained by solving problem (23) only once. However, the enhanced Algorithm 1 with the reweighting procedure will involve two loops, which results in a high computational complexity. As a result, the tradeoff between complexity and accuracy should be carefully considered in the implementation.
2) Parallelized Implementation: Each step of Algorithm 1 and Algorithm 2 can be carried out in a parallel manner. Except for the MSE weights $\boldsymbol{\alpha}$ and the Lagrangian dual variables $\mathbf{y}$, the computation for beamformer $\tilde{\mathbf{v}}_{k}$ and beamformer $\mathbf{w}_{i}$ can be performed in the parallel computing units of BBU pool for each RRH and each UE, respectively, without any information exchange. After that, both $\boldsymbol{\alpha}$ and $\mathbf{y}$ are updated with outputted $\tilde{\mathbf{v}}_{k}$ and $\mathbf{w}_{i}$. Once the update is done, $\tilde{\mathbf{v}}_{k}$ and $\mathbf{w}_{i}$ can be calculated simultaneously again in the parallel computing units of BBU pool.

3) Computational Complexity: The proposed solution is highly efficient as each step of Algorithm 1 and Algorithm 2 is in closed-form. Specifically, the main computational complexity is related to the matrix inversion in (20) and (38), which have computational complexity in the order of $\mathcal{O}\left(N^{3}\right)$ and $\mathcal{O}\left((M K)^{3}\right)$, respectively. Compared with the standard interior point method, which has a computational complexity in the order of $\mathcal{O}\left((M K I)^{3.5}\right)$, the proposed GSB-based solution has a lower computational complexity, especially for large-scale C-RANs.

\section{RiP-Based Equivalent Penalized WMMSE ALGORITHM}

In this section, we will use the RIP approach to solve the optimization problem in (14).

\section{A. Mixed Integer Programming Formulation}

A set of binary variables $\mathbf{s}=\left\{s_{k}: s_{k} \in\{0,1\}, k \in \mathcal{K}\right\}$ is first introduced to indicate the active/sleeping states of each RRH, where $s_{k}=1$ when RRH $k$ is activated, and $s_{k}=0$ when RRH $k$ is asleep. With $\mathbf{s}(t)$, the network power consumption model (4) can be rewritten as

$$
p(\mathbf{s}(t), \mathbf{w}(t))=\sum_{k \in \mathcal{K}} s_{k}(t)\left(\left\|\tilde{\mathbf{w}}_{k}(t)\right\|_{2}^{2}+P_{k}^{c}\right) .
$$

Replacing the penalty term of (14) with the power consumption model defined in 39, we have the following penalized weighted sum rate maximization problem:

$$
\begin{aligned}
& \max _{\mathbf{s}, \mathbf{w}} \cdot \sum_{i \in \mathcal{I}} Q_{i} R_{i}-\varphi \sum_{k \in \mathcal{K}} s_{k}\left(\left\|\tilde{\mathbf{w}}_{k}\right\|_{2}^{2}+P_{k}^{c}\right), \\
& \text { s.t. }\left\|\tilde{\mathbf{w}}_{k}\right\|_{2}^{2} \leq P_{k}, \\
& \quad s_{k}=\{0,1\},
\end{aligned}
$$

where $\varphi=V /\left(W \tau \log _{2}^{e}\right)$. As can be seen, the nonlinear crossmultiplication terms (NCMTs) in the cost function impose a great challenge on algorithm design. Inspired by the fact that $\tilde{\mathbf{w}}_{k}$ is equal to $\mathbf{0}$ if RRH $k$ is turned off, we can cancel all the NCMTs in the objective function and constraint as follows:

$$
\begin{aligned}
& \max _{\mathbf{w}, \mathbf{s}} \sum_{i \in \mathcal{I}} Q_{i} R_{i}-\varphi \sum_{k \in \mathcal{K}}\left(\left\|\tilde{\mathbf{w}}_{k}\right\|_{2}^{2}+s_{k} P_{k}^{c}\right), \\
& \text { s.t. }\left\|\tilde{\mathbf{w}}_{k}\right\|_{2}^{2} \leq s_{k} P_{k}, \\
& \quad s_{k}=\{0,1\},
\end{aligned}
$$

The above problem is a mixed integer nonlinear programming, and some standard algorithms have been developed to solve it, e.g., the branch-and-bound $(\mathrm{BnB})$ algorithm [35]. However, the computational complexity of $\mathrm{BnB}$ is prohibitive for a large-scale C-RAN. For the worst case, $2^{K}$ iterations is 
required, thus their computational complexity is approximated as $\mathcal{O}\left(2^{K}(K M I)^{3.5}\right)$, which grows exponentially with the number of RRHs and cannot be applied in practice. Efficient algorithm will be studied in the following subsection.

\section{B. Equivalent Formulation with Relaxed Integer Programming}

Utilizing the established equivalence between penalized weighted sum rate maximization and penalized WMMSE in section IV, and relaxing the binary variable $s_{k}$ to take continuous value in $[0,1]$, the problem can be transformed as:

$$
\begin{aligned}
& \min _{\boldsymbol{\alpha}, \mathbf{u}, \mathbf{s}, \mathbf{w}} \sum_{i \in \mathcal{I}} Q_{i}\left(\alpha_{i} e_{i}-\log \alpha_{i}\right)+\varphi \sum_{k \in \mathcal{K}}\left(\left\|\tilde{\mathbf{w}}_{k}\right\|_{2}^{2}+s_{k} P_{k}^{c}\right), \\
& \text { s.t. }\left\|\tilde{\mathbf{w}}_{k}\right\|_{2}^{2} \leq s_{k} P_{k}, \\
& \quad s_{k} \in[0,1] .
\end{aligned}
$$

The above problem is strongly convex for individual variable $\boldsymbol{\alpha}$ and $\mathbf{u}$ when fixing the rest. Correspondingly, the unique optimal solutions $\boldsymbol{\alpha}^{*}$ and $\mathbf{u}^{*}$ are given by (20) and (22), respectively. Furthermore, when fixing $\alpha$ and $\mathbf{u}$, the optimization problem for $\mathbf{s}$ and $\mathbf{w}$ is convex and is given by

$$
\begin{aligned}
& \min _{\mathbf{w}, \mathbf{s}} \cdot \sum_{i \in \mathcal{I}} \mathbf{w}_{i}^{H} \mathbf{C w}_{i}-2 \sum_{i \in \mathcal{I}} \operatorname{Re}\left\{\mathbf{d}_{i}^{H} \mathbf{w}_{i}\right\}+\varphi \sum_{k \in \mathcal{K}}\left(s_{k} P_{k}^{c}+\left\|\tilde{\mathbf{w}}_{k}\right\|_{2}^{2}\right), \\
& \text { s.t. }\left\|\tilde{\mathbf{w}}_{k}\right\|_{2}^{2} \leq s_{k} P_{k}, \\
& \quad s_{k} \in[0,1] .
\end{aligned}
$$

Therefore, a stationary solution can be obtained with the BCD method by iteratively optimizing over three block variables $\boldsymbol{\alpha}, \mathbf{u}$ and $\{\mathbf{s}, \mathbf{w}\}$. The algorithm is summarized in Algorithm 3

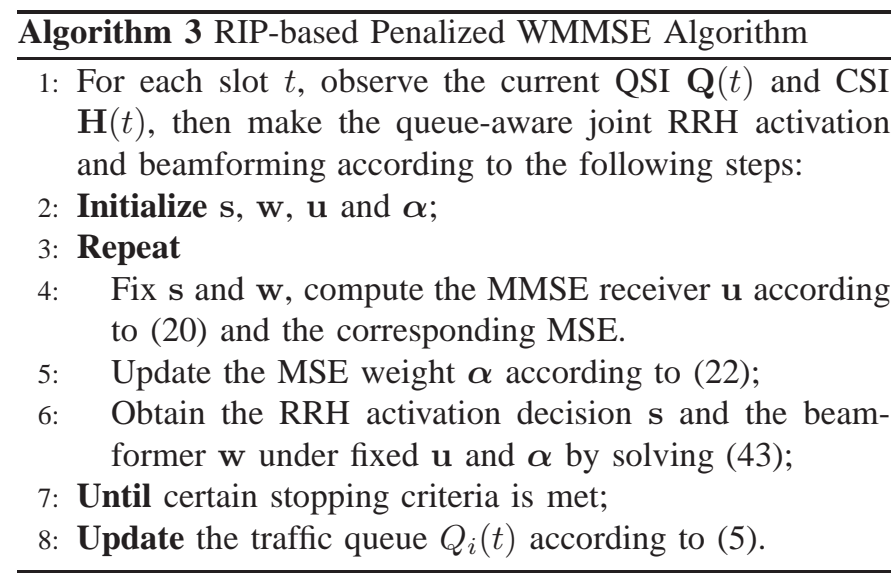

\section{RRH Activation and Beamforming based on Lagrangian Dual Decomposition}

The Lagrangian function of problem (43) is given by [36]

$$
\begin{aligned}
& L(\mathbf{w}, \mathbf{s}, \boldsymbol{\theta})=\sum_{i \in \mathcal{I}} \mathbf{w}_{i}^{H} \mathbf{C} \mathbf{w}_{i}-2 \sum_{i \in \mathcal{I}} \operatorname{Re}\left\{\mathbf{d}_{i}^{H} \mathbf{w}_{i}\right\} \\
& +\sum_{k \in \mathcal{K}}\left(\varphi P_{k}^{c}-\theta_{k} P_{k}\right) s_{k}+\sum_{k \in \mathcal{K}}\left(\varphi+\theta_{k}\right)\left\|\tilde{\mathbf{w}}_{k}\right\|_{2}^{2} .
\end{aligned}
$$

where $\boldsymbol{\theta}=\left[\theta_{1}, \theta_{2}, \ldots, \theta_{K}\right] \succeq \mathbf{0}$ is the vector of dual variables associated with the network power consumption constraints. Correspondingly, the Lagrange dual function is given by

$$
\begin{array}{r}
D(\boldsymbol{\theta})=\min _{\mathbf{s}, \mathbf{w}} . \\
\text { s.t. } s_{k} \in[0,1],
\end{array}
$$

and the dual optimization problem is formulated as

$$
\begin{gathered}
\max _{\boldsymbol{\theta}} . \quad D(\boldsymbol{\theta}), \\
\text { s.t. } \boldsymbol{\theta} \succeq \mathbf{0} .
\end{gathered}
$$

The Lagrangian function $L(\mathbf{w}, \mathbf{s}, \boldsymbol{\theta})$ is linear with $\boldsymbol{\theta}$ for any fixed $\mathbf{s}$ and $\mathbf{w}$, while the dual function $D(\boldsymbol{\theta})$ is the maximum of these linear functions. Therefore, the dual optimization problem is always concave.

Given Lagrangian dual variables, the problem of RRH activation and beamforming can be decomposed and solved separately. For the problem of RRH activation, it can be further decomposed into $K$ independent problems and solved in a parallel manner. The activation problem for RRH $k$ is given by

$$
\begin{aligned}
& \min _{s_{k}} .\left(\varphi P_{k}^{c}-\theta_{k} P_{k}\right) s_{k}, \\
& \text { s.t. } 0 \leq s_{k} \leq 1 .
\end{aligned}
$$

It can be easily seen that the optimal solution of $\mathbf{s}$ is given by

$$
s_{k}^{*}= \begin{cases}0, & \theta_{k} \leq \varphi P_{k}^{c} / P_{k}, \\ 1, & \theta_{k}>\varphi P_{k}^{c} / P_{k} .\end{cases}
$$

It is worth noting that after the integer relaxation on $s_{k}(t)$, we can still obtain optimal solution that is binary. Therefore, the integer relaxation does not introduce performance loss and there is no gap with integer relaxation.

Given Lagrangian dual variables, the problem to derive beamforming vectors is given by

$$
\min _{\mathbf{w}} \cdot \sum_{i \in \mathcal{I}} \mathbf{w}_{i}^{H} \mathbf{C w}_{i}-2 \sum_{i \in \mathcal{I}} \operatorname{Re}\left\{\mathbf{d}_{i}^{H} \mathbf{w}_{i}\right\}+\sum_{k \in \mathcal{K}}\left(\varphi+\theta_{k}\right)\left\|\tilde{\mathbf{w}}_{k}\right\|_{2}^{2},
$$

which can be further rewritten as

$$
\min _{\mathbf{w}} \cdot \sum_{i \in \mathcal{I}} \mathbf{w}_{i}^{H} \mathbf{C} \mathbf{w}_{i}-2 \sum_{i \in \mathcal{I}} \operatorname{Re}\left\{\mathbf{d}_{i}^{H} \mathbf{w}_{i}\right\}+\sum_{i \in \mathcal{I}} \mathbf{w}_{i}^{H} \boldsymbol{\Omega} \mathbf{w}_{i},
$$

where $\boldsymbol{\Omega}=\operatorname{diag}\left(\left[\varphi+\theta_{1}, \ldots, \varphi+\theta_{K}\right] \otimes \mathbf{1}_{M}\right)$ is a diagonal matrix, $\otimes$ denotes the Kronecker product of two vectors, and $\mathbf{1}_{M}$ denotes a length $M$ all-one vector. The problem (50) is an unconstrained convex problem, and it can be further decomposed into $I$ independent problems, each corresponding to an UE and solved in a parallel manner. Thus, according to the first-order optimality condition, the optimal beamforming vector for $\mathrm{UE} i$ is given by

$$
\mathbf{w}_{i}^{*}=(\mathbf{C}+\boldsymbol{\Omega})^{-1} \mathbf{d}_{i} .
$$

\section{Lagrangian Dual Variables Update}

As the dual problem is always concave w.r.t. $\theta$, we can adopt the subgradient projection method to solve it [36]. In particular, it is easy to prove that the subgradient of the dual function is obtained by

$$
\Delta \theta_{k}^{(n+1)}=\left\|\tilde{\mathbf{w}}_{k}^{(n)}\right\|_{2}^{2}-s_{k}^{(n)} P_{k},
$$

where $s_{k}^{(n)}$ is the optimal RRH activation according to (48) in the $n$-th iteration given $\boldsymbol{\theta}^{(n)}$, and $\tilde{\mathbf{w}}_{k}^{(n)}$ can be obtained from 
the optimal beamformer for each UE in the $n$-th iteration given $\boldsymbol{\theta}^{(n)}$.

Hence, with the subgradient projection method, the update equation for the dual variable $\theta_{k}$ in the $(n+1)$-th iteration is given by

$$
\theta_{k}^{(n+1)}=\theta_{k}^{(n)}+\xi^{(n+1)} \Delta \theta_{k}^{(n+1)},
$$

where $\xi^{(n+1)}$ is a sufficiently small positive step size. The whole procedure to solve (43) is summarized in Algorithm 4

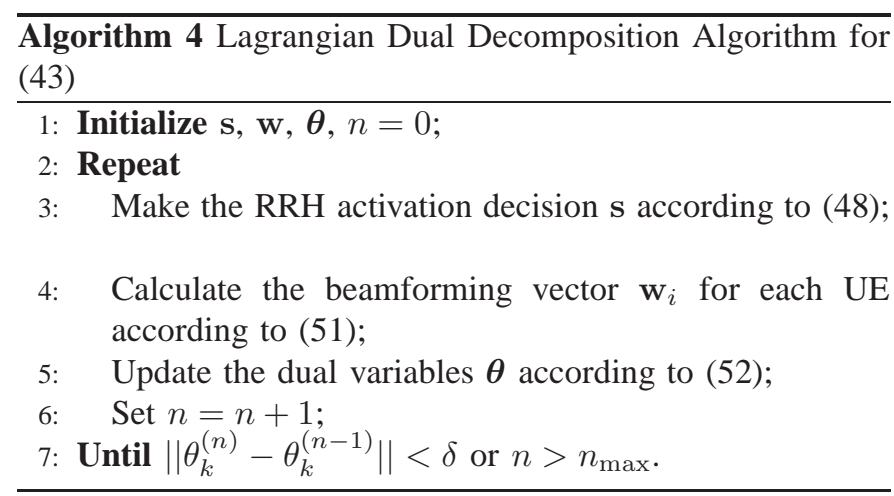

\section{E. Discussions on Implementation and Complexity}

1) Parallelized Implementation: Similarly, each step of Algorithm 3 and Algorithm 4 can be carried out in a parallel manner. Except for the MSE weights $\boldsymbol{\alpha}$ and the Lagrangian dual variables $\boldsymbol{\theta}$, the computation for RRH activation $s_{k}$ and beamformer $\mathbf{w}_{i}$ can be performed in the parallel computing units of BBU pool for each RRH and each UE, respectively, without any information exchange. After that, both $\boldsymbol{\alpha}$ and $\boldsymbol{\theta}$ are updated with outputted $s_{k}$ and $\mathbf{w}_{i}$. Once the update is done, $s_{k}$ and $\mathbf{w}_{i}$ can be calculated simultaneously again in the parallel computing units of BBU pool.

2) Computational Complexity: The proposed solution is highly efficient as each step of Algorithm 3 and Algorithm 4 is in closed-form. Specifically, the most computationally intensive operation is the matrix inversion in 20) and 51, which have computational complexity in the order of $\mathcal{O}\left(N^{3}\right)$ and $\mathcal{O}\left((M K)^{3}\right)$, respectively. Compared with the standard interior point method, which has a computational complexity in the order of $\mathcal{O}\left((M K I)^{3.5}\right)$ in our setting, the proposed RIP-based solution has a lower computational complexity, especially for large-scale C-RANs.

\section{NUMERICAL RESULTS}

In this section, we use simulation and numerical results to evaluate and compare the performance of the proposed algorithms.

\section{A. Scenarios and Parameters Setting}

The pathloss model is $127+25 \log _{10}(d)$ with $d(\mathrm{~km})$ being the propagation distance. The fast fading is modeled as independent complex Gaussian random variable distributed according to $\mathcal{C N}(0,1)$ and the noise power is $-112 \mathrm{dBm}$. We assumed that the RRHs are configured with 2 antennas, the
UEs are configured with 1 antennas, and they are uniformly and independently distributed in the square region [-500 500] $\times[-500$ 500] meters. Besides, we assume that the mean arrival rate is the same for all the UEs, i.e., $\lambda_{i}=\lambda$. We fix the power budget of each RRH as $P_{k}=2 \mathrm{~W}$. Each point of the the simulation results is averaged over 4000 slots in the simulations. Our simulations mainly compare the proposed algorithms with the full joint processing algorithm (FJP). In FJP algorithm, all the RRHs are active, and only the transmission power of RRHs is minimized by solving a penalized weighted sum rate maximization beamforming design problem based on the Lyapunov optimization. The FJP algorithm can achieve the highest cooperative beamforming gain with all the RRHs active, and the results from the FJP algorithm can serve as a delay performance lower bound of the proposed algorithms.

\section{B. System Performance versus Control Parameter $V$}

We first consider a C-RAN with $K=9$ RRHs and $I=6$ UEs. To indicate the heterogeneous power consumption of different RRHs and the fronthaul links, we set the static power consumption as $P_{k}^{c}=(2+k / 2) \mathrm{W}$ and set the drain efficiency of each RRH as $\eta_{k}=0.4$. In Fig. 2 and 3 , we evaluate the average queue delay and the average network power consumption against the control parameter $V$ when the mean arrival rate $\lambda$ is $1.25 \mathrm{Mbits} / \mathrm{slot}$ and $1.75 \mathrm{Mbits} / \mathrm{slot}$, respectively. For all the algorithms, a larger traffic mean arrival rate always results in a longer average delay and higher network power consumptions. This can be explained by the fact that more power is needed in order to timely transmit larger amount of traffic arrivals. Under a given mean arrival rate, the average network power consumption is a monotonically decreasing function in $V$. The rate of power decreasing starts to diminish with excessive increase of $V$. On the other hand, a larger $V$ can adversely affect the delay performance because the average queue length grows linearly with $V$. This is due to the fact that the system with a larger $V$ will emphasize less on delay performance but more on the network power consumption performance. Therefore the parameter $V$ features the tradeoff between power consumption and delay performance. From both figures, it is observed that GSB-based WMMSE algorithm always outperforms RIPbased WMMSE algorithm, but only by a small margin. Both algorithms achieve significant power saving compared to the FJP algorithm.

Fig. 4 shows the average number of sleeping RRHs for the two proposed algorithms against different values of the control parameter $V$. The average number of sleeping RRHs demonstrates a similar trend as the average network power consumption performance. To reduce network power consumption for a large $V$, it is necessary to turn off as many RRHs and the corresponding fronthaul links as possible, at the cost of longer average queue length.

\section{Quantitative Control of the Power-Delay Tradeoff}

The power-delay tradeoffs of various algorithms are compared in Fig. 5] The different tradeoff points are obtained 


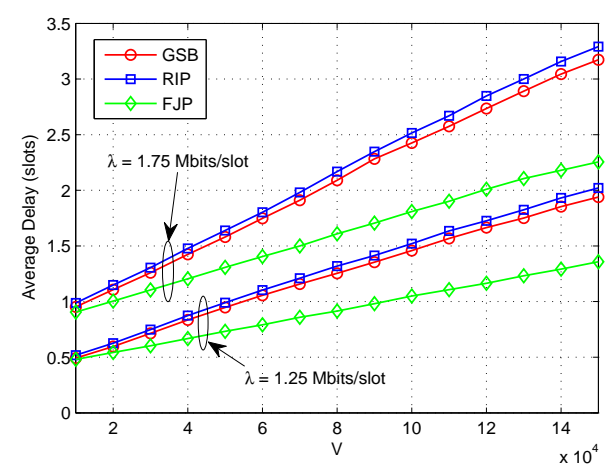

Fig. 2. Average delay vs. parameter $V$

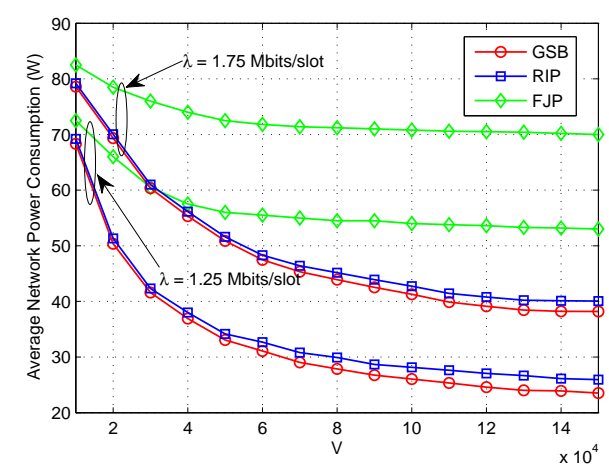

Fig. 3. Average network power consumption vs. parameter $V$

by varying the control parameter $V$. The average network power consumption is decreasing and convex in the average delay. When the delay is small, slightly increasing the delay requirement can achieve a significant amount of power saving. When the delay is excessively large, increasing the delay further results in only a very small power saving. Fig. 5 shows that GSB- and RIP-based algorithm provide significantly better power-delay tradeoff than the FJP algorithm. In addition, when the average delay decreases, the power consumption gap between the proposed algorithms and the FJP algorithm becomes smaller. This means that more RRHs need to be turned on with a stricter delay requirement. In the extreme case when all the RRHs are active, all the algorithms will perform

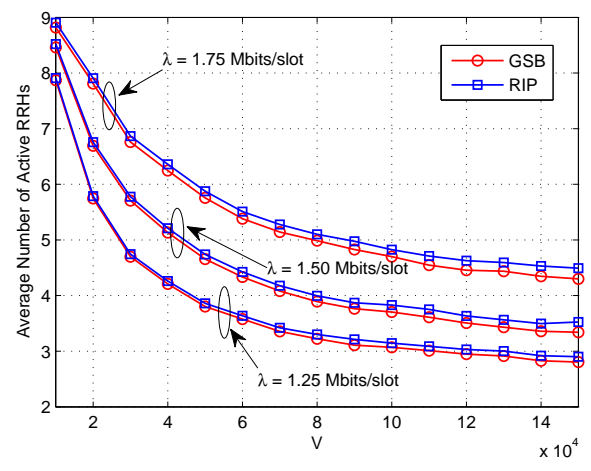

Fig. 4. Average number of active RRHs vs. parameter $V$

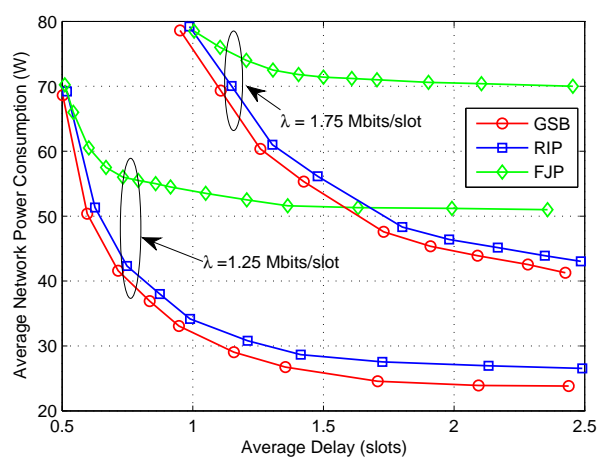

Fig. 5. Quantitative delay-power tradeoff under different $\lambda$

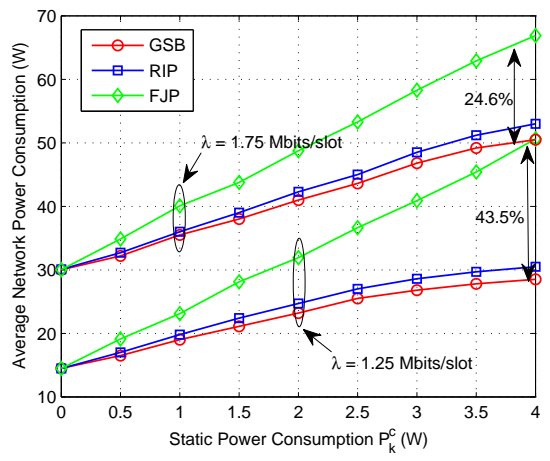

Fig. 6. Average network power consumption vs. static power consumption

FJP algorithm, yielding the same network power consumption. In the small delay regime, the proposed algorithms provide a flexible and efficient means to balance the power-delay tradeoff, given that a slight loosening of the delay requirement contributes to significant energy savings. To achieve a certain power-delay tradeoff, all we need to do is choosing an appropriate control parameter $V$.

\section{System Performance versus Static Power Consumption}

To compare the average network power consumption with different static power consumption, we set the static power consumption to be the same for all RRHs. Fig. 6 shows the average power consumption as a function of the static power consumption when the control parameter is $V=5 \times 10^{4}$. As expected, the average network power consumptions for all algorithms are increasing functions of the static power consumption. In addition, the proposed algorithms significantly outperform the FJP algorithm, especially in the high static power consumption regime. When $P_{k}^{c}=4 \mathrm{~W}$, the GSB-based algorithm achieves a power saving of $24.6 \%$ and $43.5 \%$, at $\lambda=1.75 \mathrm{Mbits} / \mathrm{slot}$ and $1.25 \mathrm{Mbits} / \mathrm{slot}$, respectively. On the other hand, the performance gap between the proposed algorithms and the FJP algorithm decreases as $P_{k}^{c}$ decreases. When $P_{k}^{c}=0$, all algorithms require almost the same network power consumption. When the static power consumption is smaller, more RRHs will be activated to achieve a higher beamforming gain. 


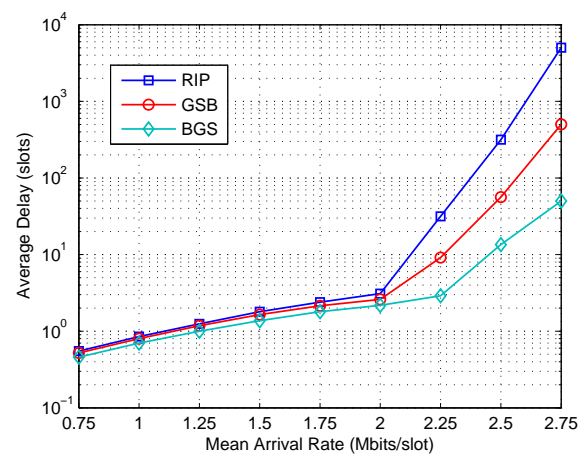

Fig. 7. Average delay vs. mean arrival rate

\section{E. System Performance versus Mean Arrival Rate}

Fig. 7 compares the average delay with different mean traffic arrival rates for the two proposed algorithms and the backward greedy selection (BGS)-based algorithm, which iteratively switches off one RRH at each step, while re-optimizing the FJP beamformer for the remaining active RRH set. The BGSbased algorithm has been shown to often yield optimal or nearoptimal solutions for RRH activation [13]. We set the control parameter as $V=8 \times 10^{4}$. It is observed that, when the mean traffic arrival rate is relatively low, the average delays of the two proposed algorithms are slightly larger than that of the BGS-based algorithm . Furthermore, the average delays for all algorithms increase sharply and tend to infinity as the mean arrival rates are beyond certain thresholds (i.e., the stability regions). Specifically, the BGS-based algorithm achieves the biggest stability region, followed by the GSB-based algorithm and the RIP-based algorithm. Therefore, congestion controls should be adopted to guarantee the queue stability when the network is with traffic load exceeding current stability regions.

\section{F. Convergence of the Algorithms}

Fig. 8 shows the average number of outer BCD iterations and the average number of inner iterations required by the GSB-based WMMSE algorithm and RIP-based WMMSE algorithm, respectively, with respect to the network scale factor $\Theta$. Here the network scale factor indicates that the considered C-RAN is with $K=9 \Theta \mathrm{RRHs}$ and $I=6 \Theta$ UEs distributed uniformly in the squared region $[-500 \sqrt{\Theta}, 500 \sqrt{\Theta}] \times$ $[-500 \sqrt{\Theta}, 500 \sqrt{\Theta}]$ meters. We can observe that both GSBbased algorithm and RIP-based algorithm can converge fairly fast under different network scale, thus both algorithms are highly scalable to large-scale C-RANs. It is observed that more iterations are required by the RIP-based solutions compared to that of GSB-based solutions. Meanwhile, as can be seen in Fig. 5, the GSB-based solution slightly outperforms the RIP-based solution. Thus, the GSB-based solution is more preferable in practice.

\section{CONCLUSION}

We have developed a joint RRH activation and beamforming algorithm for a downlink slotted C-RAN, by considering random traffic arrivals and time-varying channel fadings. The

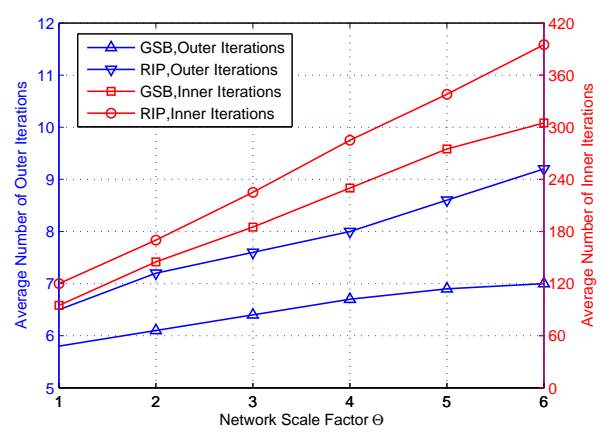

Fig. 8. Average number of iterations to reach convergence

algorithm can achieve flexible and efficient tradeoff between network power consumption and delay by adjusting a single parameter. The stochastic optimization problem of joint RRH activation and beamforming has been transformed into a penalized weighted sum rate maximization problem based on the Lyapunov optimization technique. Both GSB and RIP approaches have been used to reformulate the penalized weighted sum rate maximization problem. The corresponding algorithms for both approaches have been proposed, and they were derived based on the equivalence between the weighted sum rate maximization problem and the WMMSE problem. The algorithms are guaranteed to converge to a stationary solution. The solutions do not require any prior-knowledge of stochastic traffic arrivals and channel statistics, and can be implemented in a parallel manner. Finally, the efficiency and the efficacy of the proposed algorithms have been confirmed by the numerical simulations. For future works, it would be interesting to consider the queue-aware energy-efficient joint RRH activation and beamforming algorithms for C-RANs with imperfect channel state information (CSI) or capacity-limited fronthaul links.

\section{APPENDIX A}

\section{PRoOF OF LEMMA 1}

By leveraging on the fact that $(\max [a-b, 0]+c)^{2} \leq a^{2}+$ $b^{2}+c^{2}-2 a(b-c), \forall a, b, c \geq 0$ and squaring Eq. (5), we have $Q_{i}^{2}(t+1)-Q_{i}^{2}(t) \leq \mu_{i}^{2}(t)+A_{i}^{2}(t)-2 Q_{i}(t)\left(\mu_{i}(t)-A_{i}(t)\right)$,

According to the definition of the Lyapunov drift function, we then have the following expression by summing over all $I$ inequalities in (54) and taking expectation over both sides,

$$
\begin{aligned}
\Delta(\mathbf{Q}(t)) & \leq \frac{1}{2} \mathbb{E}\left[\sum_{i \in \mathcal{I}} \mu_{i}^{2}(t)+A_{i}^{2}(t) \mid \mathbf{Q}(t)\right] \\
& -\sum_{i \in \mathcal{I}} Q_{i}(t) \mathbb{E}\left[\mu_{i}(t)-A_{i}(t) \mid \mathbf{Q}(t)\right] .
\end{aligned}
$$

Let $B \geq \frac{1}{2} \sum_{i=1}^{I} \mathbb{E}\left[A_{i}^{2}(t)+\mu_{i}^{2}(t) \mid \mathbf{Q}(t)\right]$. Finally, the upper bound in $i=13$ can be obtained by adding $V \mathbb{E}[p(\mathcal{A}(t), \mathbf{w}(t)) \mid \mathbf{Q}(t)]$ to both sides of (55). 


\section{APPENDIX B \\ PROOF OF THEOREM 2}

Suppose that the traffic arrivals with mean arrival rate $\boldsymbol{\lambda}=\left(\lambda_{1}, \ldots, \lambda_{I}\right)$ is strictly interior to the stability region $\mathcal{C}$ (Definition 2) such that $\boldsymbol{\lambda}+\epsilon \mathbf{1} \in \mathcal{C}, \forall \epsilon>0$. Since channel conditions are i.i.d. over slots, according to Theorem 4.5 in [24], there exists a stationary randomized control policy that is independent of $\mathbf{Q}(t)$ and yields

$$
\begin{aligned}
& \mathbb{E}\left[\mu_{i}(t) \mid \mathbf{Q}(t)\right]=\mathbb{E}\left[\mu_{i}(t)\right] \geq \lambda_{i}+\epsilon, \forall i, \\
& \mathbb{E}[p(\mathcal{A}(t), \mathbf{w}(t)) \mid \mathbf{Q}(t)]=\mathbb{E}[p(\mathcal{A}(t), \mathbf{w}(t))]=\bar{p}(\epsilon) .
\end{aligned}
$$

As the stationary randomized control policy is simply a particular control policy, it certainly satisfies 13) in Lemma 1. In addition, since (14) is obtained by minimizing the right-handside (R.H.S.) of 13 among all feasible policies (including the stationary randomized control policy), by combining (56) with 13, we have

$$
\begin{aligned}
& \Delta(\mathbf{Q}(t))+V \mathbb{E}[p(\mathcal{A}(t), \mathbf{w}(t)) \mid \mathbf{Q}(t)] \leq B+ \\
& V \mathbb{E}[p(\mathcal{A}(t), \mathbf{w}(t)) \mid \mathbf{Q}(t)]+\sum_{i \in \mathcal{I}} Q_{i}(t) \mathbb{E}\left[A_{i}(t)-\mu_{i}(t) \mid \mathbf{Q}(t)\right] \\
& \leq B+V \bar{p}(\epsilon)-\varepsilon \sum_{i \in \mathcal{I}} Q_{i}(t) .
\end{aligned}
$$

Using the results in the proof of Theorem 1, it follows that $\sum_{i \in \mathcal{I}} Q_{i}(t) \leq \frac{B+V \bar{p}(\epsilon)}{\epsilon}$, which proves that solving 14 optimally stabiles all the queues.

\section{APPENDIX C \\ PROOF OF PROPOSITION 1}

Let $L=K M I$ and define the index set $\mathcal{V}=\{1,2, \ldots, L\}$, then we have $\mathbf{w}=\left[w_{l}: l \in \mathcal{V}\right]$. Define the set $\mathcal{I}_{k}=$ $\{(k-1) M I+1, \ldots, k M I\}$ as a partion of $\mathcal{V}$, then we have $\tilde{\mathbf{w}}_{k}=\mathbf{w}_{\mathcal{I}_{k}}=\left[w_{l}: l \in \mathcal{I}_{k}\right]$. Furthermore, define the support of beamformer $\mathbf{w}$ as $\mathcal{T}(\mathbf{w})=\left\{l \mid w_{l} \neq 0\right\}$, then the power consumption model can be rewritten as

$$
p(\mathbf{w})=\sum_{k \in \mathcal{K}}\left(\frac{1}{\eta_{k}}\left\|\mathbf{w}_{\mathcal{I}_{k}}\right\|_{2}^{2}+P_{k}^{c} \mathbb{I}\left(\mathcal{T}(\mathbf{w}) \cap \mathcal{I}_{k} \neq \emptyset\right)\right),
$$

where $\mathbb{I}(\mathcal{E})$ is an indicator function with value 1 if the event $\mathcal{E}$ is true and 0 otherwise. To simplify notation, let $T(\mathbf{w})=$ $\sum_{k \in \mathcal{K}} \frac{1}{\eta_{k}}\left\|\mathbf{w}_{\mathcal{I}_{k}}\right\|_{2}^{2}$, and let $F(\mathcal{T}(\mathbf{w}))=P_{k}^{c} \mathbb{I}\left(\mathcal{T}(\mathbf{w}) \cap \mathcal{I}_{k} \neq \emptyset\right)$. It can been seen that $F(\cdot)$ is combinatorial in $\mathbf{w}$ and is nonconvex. We will obtain a convex relaxation of the combinatorial objective function.

We first construct the tightest positively homogeneous lower bound of $p(\mathbf{w})$, which is given by [37]

$$
\begin{aligned}
p_{h}(\mathbf{w}) & =\inf _{\phi>0} \frac{p(\phi \mathbf{w})}{\phi}=\inf _{\phi>0} \phi T(\mathbf{w})+\frac{1}{\phi} F(\mathcal{T}(\mathbf{w})) \\
& =2 \sqrt{F(\mathcal{T}(\mathbf{w})) T(\mathbf{w})} .
\end{aligned}
$$

The last equality in (59) is obtained by solving $\partial \frac{p(\phi \mathbf{w})}{\phi} / \partial \phi=$ 0 . However, $p_{h}(\mathbf{w})$ is still non-convex in $\mathbf{w}$.

We next calculate the convex envelope of $p_{h}(\mathbf{w})$. Define diagonal matrices $\boldsymbol{\Lambda} \in \mathbb{R}^{L \times L}, \boldsymbol{\Xi} \in \mathbb{R}^{L \times L}$ with the $k$-th diagonal block being $\eta_{k} \mathbf{I}_{M I}$ and $\frac{1}{\eta_{k}} \mathbf{I}_{M I}$, respectively. The Fenchel conjugate of $p_{h}(\mathbf{w})$ is given by

$$
\begin{aligned}
& p_{h}^{*}(\mathbf{z})=\sup _{\mathbf{w} \in \mathbb{C}^{L}}\left(\mathbf{z}^{T} \boldsymbol{\Lambda}^{T} \boldsymbol{\Xi} \mathbf{w}-2 \sqrt{F(\mathcal{T}(\mathbf{w})) T(\mathbf{w})}\right) \\
& =\sup _{\mathcal{X} \in \mathcal{V}} \sup _{\mathbf{w} \in \mathbb{C}|\mathcal{X}|}\left(\mathbf{z}_{\mathcal{X}}^{T} \boldsymbol{\Lambda}_{\mathcal{X}}^{T} \boldsymbol{\Xi}_{\mathcal{X}} \mathbf{w}_{\mathcal{X}}-2 \sqrt{F(\mathcal{X}) T(\mathbf{w} \mathcal{X})}\right) \\
& = \begin{cases}0, & \text { if } \hat{p}^{*}(\mathbf{z})=\sup _{\mathcal{X} \subseteq \mathcal{V}, \mathcal{X} \neq \emptyset} \frac{\left\|\mathbf{z}_{\mathcal{X}} \boldsymbol{\Lambda}_{\mathcal{X}}\right\|_{2}}{2 \sqrt{F(\mathcal{X})}} \leq 1, \\
\infty, & \text { otherwise. }\end{cases}
\end{aligned}
$$

where $\mathbf{z}_{\mathcal{X}}$ is the $|\mathcal{X}|$-dimensional vector formed with the entries of $\mathbf{z}$ indexed by $\mathcal{X}$ (similarly for $\mathbf{w}$ ), and $\boldsymbol{\Lambda}_{\mathcal{X}}$ is the $|\mathcal{X}| \times|\mathcal{X}|$-dimensional matrix formed with both the rows and the columns of $\boldsymbol{\Lambda}$ indexed by $\mathcal{X}$ (similarly for $\boldsymbol{\Xi}$ ). Consider the norm $\hat{p}(\mathbf{w})$ whose dual norm is defined as $\hat{p}^{*}(\mathbf{z})$ in Eq. [60). According to Proposition 2 in [37], $\hat{p}(\mathbf{w})$ is the convex envelope of $p(\mathbf{w})$.

Therefore, the tightest convex positively homogenous lower bound of $p(\mathbf{w})$ has the following inequality:

$$
\begin{aligned}
& \hat{p}(\mathbf{w})=\sup _{\hat{p}^{*}(\mathbf{z}) \leq 1} \mathbf{w}^{T} \mathbf{z} \leq \sup _{\hat{p}^{*}(\mathbf{z}) \leq 1} \sum_{k \in \mathcal{K}}\left\|\mathbf{w}_{\mathcal{I}_{k}}\right\|_{2}\left\|\mathbf{z}_{\mathcal{I}_{k}}\right\|_{2} \\
& \leq \sup _{\hat{p}^{*}(\mathbf{z}) \leq 1}\left(\sum_{k \in \mathcal{K}} \sqrt{\frac{P_{k}^{c}}{\eta_{k}}}\left\|\mathbf{w}_{\mathcal{I}_{k}}\right\|_{2}\right)\left(\max _{k \in \mathcal{K}} \sqrt{\frac{\eta_{k}}{P_{k}^{c}}}\left\|\mathbf{z}_{\mathcal{I}_{k}}\right\|_{2}\right) \\
& =2 \sum_{k \in \mathcal{K}} \sqrt{\frac{P_{k}^{c}}{\eta_{k}}}\left\|\mathbf{w}_{\mathcal{I}_{k}}\right\|_{2},
\end{aligned}
$$

which is obtained using the norm properties. Actually, the above inequality always holds with equality. Specifically, let $\overline{\mathbf{z}}_{\mathcal{I}_{k}}=2 \sqrt{\frac{P_{k}^{c}}{\eta_{k}}} \frac{\mathbf{w}_{\mathcal{I}_{k}}^{H}}{\left\|\mathbf{w}_{\mathcal{I}_{k}}^{H}\right\|_{2}}$ such that $\hat{p}^{*}(\overline{\mathbf{z}})=1$, then we have

$\hat{p}(\mathbf{w})=\sup _{\hat{p}^{*}(\mathbf{z}) \leq 1} \mathbf{w}^{T} \mathbf{z} \geq \sum_{k \in \mathcal{K}} \mathbf{w}_{\mathcal{I}_{k}}^{T} \overline{\mathbf{z}}_{\mathcal{I}_{k}}=2 \sum_{k \in \mathcal{K}} \sqrt{\frac{P_{k}^{c}}{\eta_{k}}}\left\|\mathbf{w}_{\mathcal{I}_{k}}\right\|_{2}$,

which is obtained using the definition of convex envelope. Therefore, we finally have $2 \sum_{k \in \mathcal{K}} \sqrt{\frac{P_{k}^{c}}{\eta_{k}}}|| \mathbf{w}_{\mathcal{I}_{k}} \|_{2} \leq \hat{p}(\mathbf{w}) \leq$ $2 \sum_{k \in \mathcal{K}} \sqrt{\frac{P_{k}^{c}}{\eta_{k}}}\left\|\mathbf{w}_{\mathcal{I}_{k}}\right\|_{2}$, i.e., 17.

\section{APPENDIX D \\ PROOF OF THEOREM 6}

By comparing problem (24) and problem 29), when $\mathbf{x}=\mathbf{v}$ and $\mathbf{z}=\mathbf{w}$, we can observe that

$$
\begin{aligned}
& f(\mathbf{x})=\sum_{k \in \mathcal{K}} \beta_{k}\left\|\tilde{\mathbf{v}}_{k}\right\|_{2}, \\
& g(\mathbf{z})=\sum_{i \in \mathcal{I}} \mathbf{w}_{i}^{H} \mathbf{C} \mathbf{w}_{i}-2 \sum_{i \in \mathcal{I}} \operatorname{Re}\left\{\mathbf{d}_{i}^{H} \mathbf{w}_{i}\right\} \\
& \mathbf{A}=\mathbf{I}, \mathbf{B}=\mathbf{I}, \mathbf{c}=\mathbf{0}, \\
& \mathcal{C}_{1}=\left(\mathbf{x} \mid\left\|\tilde{\mathbf{v}}_{k}\right\|_{2}^{2} \leq P_{k}, \forall k \in \mathcal{K}\right), \mathcal{C}_{2}=\mathbf{z} .
\end{aligned}
$$

Since $\mathbf{A}^{T} \mathbf{A}=\mathbf{I}$ and $\mathbf{B}^{T} \mathbf{B}=\mathbf{I}$ are invertible, and both $\mathcal{C}_{1}$ and $\mathcal{C}_{2}$ are convex sets, then according to Theorem 3 , we can conclude that every limit point $\mathbf{w}^{(n)}$ and $\mathbf{v}^{(n)}$ generated by Algorithm 2 is an optimal solution of problem (23). 


\section{REFERENCES}

[1] C. I, C. Rowell, S. Han, Z. Xu, G. Li, and Z. Pan, "Toward green and soft: a 5G perspective," IEEE Commun. Mag., vol. 52, no. 2, pp. 66-73, Feb. 2014.

[2] China Mobile Research Institute, "C-RAN: The road towards green RAN," White Paper, ver. 3.0, Dec. 2013.

[3] P. Rost, C. J. Bernardos, A. D. Domenico, M. D. Girolamo, M. Lalam, A. Maeder, D. Sabella, and D. Wübben, "Cloud technologies for flexible 5G radio access networks," IEEE Commun. Mag., vol. 52, no. 5, pp. 68-76, May 2014.

[4] NGMN alliance, "Suggestion on potential solution to C-RAN," Jan. 2013.

[5] C. Wang, F. Haider, X. Gao, X. You, Y. Yang, D. Yuan, H. M. Aggoune, H. Haas, S. Fletcher, and E. Hepsaydir, "Cellular architecture and key technologies for $5 \mathrm{G}$ wireless communication networks," IEEE Commun. Mag., vol. 52, no. 2, pp. 122-130, Feb. 2014.

[6] J. Zhao, T. Q. S. Quek, and Z. Lei, "Coordianted multipoint transmission with limited backhaul data transfer," IEEE Trans Wireless Commun., vol. 12, no. 6, pp. 2762-2774, Jun. 2013.

[7] F. Zhuang and V. K. N. Lau, "Backhaul limited asymmetric cooperation for MIMO cellular networks via semidefinite relaxation," IEEE Trans. Signal Process., vol. 62, no. 3, pp. 684-693, Feb. 2014.

[8] V. N. Ha, L. B. Le, and N. D. Dao, "Coordinated multipoint (CoMP) transmission design for Cloud-RANs with limited fronthaul capacity constraints," IEEE Trans. Veh. Tech., early access, 2015.

[9] B. Dai and W. Yu, "Sparse beamforming and user-centric clustering for downlink cloud radio access network," IEEE Access, vol. 2, pp. 13261339, Oct. 2014.

[10] Y. Cheng, M. Pesavento, and A. Philipp, "Joint network optimization and downlink beamforming for CoMP transmissions using mixed integer conic programming," IEEE Trans. Signal Process., vol. 61, pp. 39723987, Aug. 2013.

[11] S. Luo, R. Zhang, and T. J. Lim, "Downlink and uplink energy minimization through user association and beamforming in cloud RAN," IEEE Trans. Wireless Commun., vol. 14, no. 1, pp. 494-508, Jan. 2015.

[12] S. Tombaz, P. Monti, K. Wang, A. Västberg, M. Forzati, and J. Zander, "Impact of backhauling power consumption on the deployment of heterogeneous mobile networks," in Proc. IEEE Global Commun. Conf. (GLOBECOM), Houston, TX, USA, Dec. 2011, pp. 1-5.

[13] Y. Shi, J. Zhang, and K. B. Letaief, "Group sparse beamforming for green cloud-RAN," IEEE Trans Wireless Commun., vol. 13, no. 5, pp. 2809-2823, May 2014.

[14] B. Dai and W Yu, "Energy efficiency of downlink transmission strategies for cloud radio access networks," IEEE J. Sel. Areas Commn., Availabe: http://arxiv.org/abs/1601.01070v2

[15] Y. Cui, V. K. N. Lau, R. Wang, H. Huang, and S. Zhang, "A survey on delay-aware resource control for wireless systems - large deviation theory, stochastic Lyapunov drift and distributed stochastic learning," IEEE Trans. Inf. Theory, vol. 58, no. 3, pp. 1677-1701, Mar. 2012.

[16] M. J. Neely, E. Modiano, and C. Li, "Fairness and optimal stochastic control for heterogeneous networks," IEEE/ACM Trans. Netw., vol. 16, no. 2, pp. 396-409, Apr. 2008.

[17] L. B. Le, E. Modiano, and N. B. Shroff, "Optimal control of wireless networks with finite buffers," IEEE/ACM Trans. Netw., vol. 20, no. 4, pp. 1316-1329, Aug. 2012.

[18] M. J. Neely, "Delay analysis for maximal scheduling with flow control in wireless networks with bursty traffic," IEEE/ACM Trans. Netw., vol. 17, no. 4, pp. 1146-1159, Aug. 2009.

[19] M. J. Neely, "Energy optimal control for time-varying wireless networks," IEEE Trans. Inf. Theory, vol. 52. no. 7, pp. 2915-2934, Jul. 2006.

[20] H. Ju, B. Liang, J. Li, and X. Yang, "Dynamic power allocation for throughput utility maximization in interference-limited networks," IEEE Wireless Commun. Lett., vol. 2, no. 1, pp. 22-25, Jan. 2013.

[21] S. Park, O. Simeone, O. Sahin, and S. Shamai, "Joint precoding and multivariate backhaul compression for the downlink of cloud radio access networks," IEEE Trans. Signal Process., vol. 61, no. 22, pp. 5646-5658, Nov. 2013.

[22] G. Auer, V. Giannini, C. Desset, I. Godor, P. Skillermark, M. Olsson, M. A. Imran, D. Sabella, M. J. Gonzalez, O. Blume, and A. Fehske, "How much energy is needed to run a wireless network?" IEEE Wireless Commun., vol. 18, pp. 40-49, Oct. 2011.

[23] Y. A. Sambo, M. Z. Shakir, K. A. Qaraqe, E. Serpedin, M. A. Imran, "Expanding cellular coverage via cell-edge deployment in heterogeneous networks: spectral efficiency and backhaul power consumption perspectives," IEEE Commun. Mag., vol. 52, no. 6, pp. 140-149, Jun. 2014.
[24] M. J. Neely, Stochastic Network Optimization with Application to Communication and Queueing Systems, Morgan\&Claypool., 2010.

[25] M. J. Neely, "Delay-based network uility maximization," IEEE/ACM Trans. Netw., vol. 21. no. 1, pp. 41-54, Feb. 2013.

[26] Z. Q. Luo and S. Zhang, "Dynamic spectrum management: Complexity and duality," IEEE J. Sel. Areas Signal Process., vol. 2, no. 1, pp. 57-73, Feb. 2008.

[27] J. Li, J. Wu, M. Peng, W. Wang, and V. K. N. Lau, "Queue-aware joint remote radio head activation and beamforming for green cloud radio access networks," in Proc. IEEE Global Commun. Conf. (GLOBECOM), San Diego, CA, USA, Dec. 2015.

[28] Y. C. Eldar and G. Kutyniok, Compressed Sensing: Theory and Applications, Cambridge, U.K.: Cambridge Univ. Press, 2012.

[29] E. Candes and T. Tao, "Near-optimal signal recovery from random projections: Universal encoding strategies?" IEEE Trans. Inf. Theory, vol. 52, no. 12 , pp. 5406-5425, Dec. 2006.

[30] S. S. Christensen, R. Agarwal, E. Carvalho, and J. M. Cioffi, "Weighted sum-rate maximization using weighted MMSE for MIMO-BC beamforming design," IEEE Trans Wireless Commun., vol. 7, no. 12, pp. 4792-4799, Dec. 2008.

[31] Q. Shi, M. Razaviyayn, Z. Luo, and C. He, "An iteratively weighted MMSE approach to distributed sum-utility maximization for a MIMO interfering broadcast channel," IEEE Trans. Signal Process., vol. 59, no. 9, pp. 4331-4340, Sep. 2011

[32] W. Deng, W. Yin, and Y. Zhang, "Group sparse optimization by alternating direction method," Technical Report, Rice University, 2011.

[33] S. Boyd, N. Parikh, E. Chu, B. Peleato, and J. Eckstein, "Distributed optimization and statistical learning via the alternating direction method of multipliers," Found. Trends Mach. Learn., vol. 3, no. 1, pp. 1-122, 2011.

[34] F. Bach, R. Jenatton, J. Mairal, and G. Obozinski, "Convex optimization with sparsity-inducing norms," in Optimization for Machine Learning, S. Sra, S. Nowozin, and S. J. Wright, Eds. Cambridge, MA: MIT Press, 2011.

[35] D. Wei and A. V. Oppenheim, "A branch-and-bound algorithm for quadratically-constrained sparse filter design," IEEE Trans. Signal Process., vol. 61, no. 4, pp. 1006-1018, Feb. 2013.

[36] S. Boyd and L. Vandenberghe, Convex Optimization, Cambridge, U.K.: Cambridge University Press, 2004.

[37] G. Obozinski and F. Bach, "Convex relaxation for combinatorial penalties," a Technical Report 00694765, HAL, 2012.

[38] P. Teseng, "Convergence of a block coordinate descent method for nondifferentiable minimization," J. Opt. Theory and App., vol. 109, no. 3, pp. 475-494, Jun. 2001. 